\title{
Leading asymptotic terms of the three-body Coulomb scattering wave function
}

\author{
A. M. Mukhamedzhanov, ${ }^{1}$ A. S. Kadyrov, ${ }^{2}$ and F. Pirlepesov ${ }^{1}$ \\ ${ }^{1}$ Cyclotron Institute, Texas A\&M University, College Station, Texas 77843, USA \\ ${ }^{2}$ Centre for Atomic, Molecular and Surface Physics, Division of Science and Engineering, Murdoch University, Perth 6150, Australia
}

(Received 31 August 2005; published 18 January 2006)

\begin{abstract}
The asymptotic wave function derived by Alt and Mukhamedzhanov [Phys. Rev. A 47, 2004 (1993)] and Mukhamedzhanov and Lieber [Phys. Rev. A 54, 3078 (1996)] has been refined in the region where the pair $(\beta, \gamma)$ remains close to each other while the third particle $\alpha$ is far away from them $\left(\rho_{\alpha} \rightarrow \infty, r_{\alpha} / \rho_{\alpha} \rightarrow 0\right)$. The improved wave function satisfies the Schrödinger equation up to the terms of order $O\left(1 / \rho_{\alpha}^{3}\right)$, provides the leading asymptotic terms of the three-body scattering wave function with Coulomb interactions, and gives further insight into the continuum behavior of the three-charged-particle wave function, and helps to obtain the $3 \rightarrow 3$ scattered wave. This opens up further ways of solving and analyzing the three-body Schrödinger equation by numerical means.
\end{abstract}

DOI: 10.1103/PhysRevA.73.012713

PACS number(s): 03.65.Nk, 21.45.+v, 34.10.+x

\section{INTRODUCTION}

The quantum dynamics of three charged particles is described by Schrödinger's equation, which should be supplemented by proper boundary conditions. Merkuriev and Faddeev [1] claimed that the solution of this equation exists and is unique if the boundary conditions are known in all asymptotic regions. There are two types of three-body scattering wave functions. The first type evolves from an initial three-body incident wave describing three incident particles in continuum. The second type of the three-body scattering wave function evolves from a two-body incident wave corresponding to a collision of a two-body bound state with a third particle.

The three-body incident wave represents the leading asymptotic terms of the total three-body scattering wave function [1-3]. The knowledge of the three-body incident wave is important for many reasons: as a leading term of the three-body wave function it can be used in calculations of the breakup matrix elements if the kinematics is such that the asymptotic region gives the leading contribution; the knowledge of the three-body incident wave is necessary in direct solution of the three-body Schrödinger equation for the scattering wave function of the first type. The asymptotic behavior of the three-body incident wave depends on the asymptotic region under consideration. In the asymptotic region where two particles, for example, $\beta$ and $\gamma$, are close to each other and far away from the third particle $\alpha$, the threebody incident wave can be written as an asymptotic series in powers $1 / \rho_{\alpha}$, where $\rho_{\alpha}$ is the distance between the center of mass of the system $(\beta, \gamma)$ and the third particle $\alpha$. The leading asymptotic terms $O(1)$ and $O\left(1 / \rho_{\alpha}\right)$ of the three-body incident wave for charged particles have been obtained analytically in $[2,3]$. These asymptotic terms satisfy the Schrödinger equation up to $O\left(1 / \rho_{\alpha}^{2}\right)$. In this work we will derive all the leading asymptotic terms of the three-chargedparticle incident wave of order $O\left(1 / \rho_{\alpha}^{2}\right)$. Combined with the previously derived terms of order $O(1)$ and $O\left(1 / \rho_{\alpha}\right)$ [2,3], they provide the asymptotic solution of the Schrödinger equation for three charged particles in continuum up to terms
$O\left(1 / \rho_{\alpha}^{3}\right)$. The terms $O\left(1 / \rho_{\alpha}^{2}\right)$ satisfy first order differential equations. It is worth mentioning that the terms $O\left(1 / \rho_{\alpha}^{3}\right)$ are the next order terms compared to the three-body scattered wave, which is $O\left(1 / R^{5 / 2}\right)$, where $R$ is the hyperradius. This term, as well as the leading-order asymptotic terms of the three-body wave function of the second type have been given in Refs. $[4,5]$. Practical ways of extracting the scattering and breakup amplitudes using these asymptotic wave functions have been presented in Refs. [6,7].

The paper is organized in the following way. In Sec. I we introduce the three-body nomenclature and give the statement of the problem. In Sec. II we recall some of the important relations relevant to two-body scattering. In Secs. III-V we present asymptotic solutions of the three-body Schrödinger equation in all orders which can be obtained analytically with the asymptotic method. Finally, Sec. VI concludes the paper.

\section{STATEMENT OF THE PROBLEM}

We consider a nonrelativistic three-body problem for charged particles of mass $m_{\alpha}$ and charge $z_{\alpha}, \alpha=1,2,3$, in the continuum state. We follow the notations used in Ref. [2]. The greek letters stand for constituent particles of the threebody system or for the pair of two other particles. For example, $\alpha$ labels the particle or the pair $\beta+\gamma$. Such a supplemental notation is customary in few-body physics. The following conventional notations for the two-body quantities are also used: $A_{\alpha} \equiv A_{\beta \gamma}$, where $\alpha \neq \beta \neq \gamma$. The Jacobi coordinates are determined as follows: $\mathbf{r}_{\alpha}$ is the relative coordinate between particles $\beta$ and $\gamma$, and $\mathbf{k}_{\alpha}$ is its canonically conjugated momentum. $\mu_{\alpha}=m_{\beta} m_{\gamma} / m_{\beta \gamma}$ is their reduced mass, $m_{\beta \gamma}=m_{\beta}+m_{\gamma}$. Similarly, $\boldsymbol{\rho}_{\alpha}$ is the relative coordinate between the center of mass of the pair $(\beta, \gamma)$ and particle $\alpha$, and $\mathbf{q}_{\alpha}$ is its canonically conjugated relative momentum. $M_{\alpha}=m_{\alpha} m_{\beta \gamma} / M, M=\sum_{\nu=1}^{3} m_{\nu}$ is total mass of the three-body system. There are three sets of Jacobi coordinates $\mathbf{r}_{\nu}, \boldsymbol{\rho}_{\nu}$, where $\nu=\alpha, \beta, \gamma$. We frequently need the relations between the coordinates, and conjugate momenta for a channel 
$\nu=\beta, \gamma$ and the corresponding $\alpha$-channel variables. They are given by the following relations:

$$
\begin{aligned}
& \left(\begin{array}{l}
\boldsymbol{\rho}_{\nu} \\
\mathbf{r}_{\nu}
\end{array}\right)=\left(\begin{array}{cc}
-\frac{m_{\alpha}}{M-m_{\nu}} & \epsilon_{\nu \alpha} \frac{\mu_{\nu}}{M_{\alpha}} \\
-\epsilon_{\nu \alpha} & -\frac{m_{\nu}}{m_{\beta \gamma}}
\end{array}\right)\left(\begin{array}{l}
\boldsymbol{\rho}_{\alpha} \\
\mathbf{r}_{\alpha}
\end{array}\right), \\
& \left(\begin{array}{l}
\mathbf{q}_{\nu} \\
\mathbf{k}_{\nu}
\end{array}\right)=\left(\begin{array}{cc}
-\frac{m_{\nu}}{m_{\beta \gamma}} & \epsilon_{\nu \alpha} \\
-\epsilon_{\nu \alpha} \frac{\mu_{\alpha}}{M_{\nu}} & -\frac{m_{\alpha}}{M-m_{\nu}}
\end{array}\right)\left(\begin{array}{l}
\mathbf{q}_{\alpha} \\
\mathbf{k}_{\alpha}
\end{array}\right),
\end{aligned}
$$

where $\nu=\beta, \gamma$ and the antisymmetric symbol $\epsilon_{\alpha \nu}=-\epsilon_{\nu \alpha}$, with $\epsilon_{\alpha \nu}=1$ for $(\alpha, \nu)$ being a cyclic permutation of $(1,2,3)$, and $\epsilon_{\alpha \alpha}=0$. The motion of the three particles is described by the Schrödinger equation in the configuration space

$$
\left\{E-T_{\mathbf{r}_{\alpha}}-T_{\boldsymbol{\rho}_{\alpha}}-V\right\} \Psi_{\mathbf{k}_{\alpha} \mathbf{q}_{\alpha}}^{(+)}\left(\mathbf{r}_{\alpha}, \boldsymbol{\rho}_{\alpha}\right)=0
$$

where $V=\sum_{\nu=1}^{3} V_{\nu}, V_{\nu}=V_{\nu}^{C}\left(\mathbf{r}_{\nu}\right)+V_{\nu}^{N}\left(\mathbf{r}_{\nu}\right)$. The Coulomb potential is given by $V_{\alpha}^{C}\left(\mathbf{r}_{\alpha}\right)=Z_{\beta} Z_{\gamma} e^{2} / r_{\alpha}, Z_{\nu} e$ is the charge of particle $\nu$. Similarly, $V_{\nu}^{N}$ is the nuclear potential between the particles of the $\nu$ pair, where $\nu=\alpha, \beta, \gamma . T_{\mathbf{r}_{\alpha}}=-\Delta_{\mathbf{r}_{\alpha}} / 2 \mu_{\alpha}$ is the kinetic energy operator for the relative motion of particles $\beta$ and $\gamma$, and $T_{\boldsymbol{\rho}_{\alpha}}=-\Delta_{\boldsymbol{\rho}_{\alpha}} / 2 M_{\alpha}$ is the kinetic energy operator for the relative motion of particle $\alpha$ and the center of mass of the pair $(\beta, \gamma)$, respectively.

Our aim is to derive the asymptotic behavior of the threebody incident wave up to terms $O\left(1 / \rho_{\alpha}^{3}\right)$ in the asymptotic region $\Omega_{\alpha}$, where $r_{\alpha} / \rho_{\alpha} \rightarrow 0$ and $\rho_{\alpha} \rightarrow \infty$. This incident wave provides the leading asymptotic terms of the three-body scattering wave function of the first type. General asymptotic behavior of the three-body scattering wave function is given by $[1]$

$$
\begin{aligned}
\Psi_{\mathbf{k}_{\alpha^{\prime}} \mathbf{q}_{\alpha}}^{(+)} \approx & \widetilde{\Psi}_{\mathbf{k}_{\alpha^{\prime}} \mathbf{q}_{\alpha}}^{(+)}+\sum_{\nu=\alpha, \beta, \gamma} \varphi_{\nu}\left(\mathbf{r}_{\nu}\right) \frac{\mathcal{M}_{3 \rightarrow 2}^{(\nu)}}{\rho_{\nu}} e^{i q_{\nu} \rho_{\nu}-i \bar{\eta}_{\nu} \ln \left(2 q_{\nu} \rho_{\nu}\right)} \\
& +\frac{\mathcal{M}_{3 \rightarrow 3}}{R^{5 / 2}} e^{i \kappa R-i \lambda_{0} \ln (2 \kappa R)} .
\end{aligned}
$$

Here the first term is the incident three-body wave, the sum over $\nu$ provides the two-body outgoing scattered waves and corresponds to the $3 \rightarrow 2$ processes. The last term descibes the outgoing three-body scattered wave. Also, $\bar{\eta}_{\alpha}=\left(Z_{\beta}\right.$ $\left.+Z_{\gamma}\right) Z_{\alpha} e^{2} M_{\alpha} / q_{\alpha}$ is the Coulomb parameter for the Coulomb interaction between particles $\alpha$ and the center of mass of the system $\beta+\gamma$; the Coulomb parameter $\lambda_{0}$ is determined in Ref. [5]. We use the system of units such that $\hbar=c=1$. Formally we can determine the incident wave as an asymptotic difference

$$
\begin{aligned}
\widetilde{\Psi}_{\mathbf{k}_{\alpha}, \mathbf{q}_{\alpha}}^{(+)} \approx & \Psi_{\mathbf{k}_{\alpha}, \mathbf{q}_{\alpha}}^{(+)}-\sum_{\nu=\alpha, \beta, \gamma} \varphi_{\nu}\left(\mathbf{r}_{\nu}\right) \frac{\mathcal{M}_{3 \rightarrow 2}^{(\nu)}}{\rho_{\nu}} e^{i q_{\nu} \rho_{\nu}-i \bar{\eta}_{\nu} \ln \left(2 q_{\nu} \rho_{\nu}\right)} \\
& -\frac{\mathcal{M}_{3 \rightarrow 3}}{R^{5 / 2}} e^{i \kappa R-i \lambda_{0} \ln (2 \kappa R)}
\end{aligned}
$$

From this equation it is clear that the three-body incident wave is a part of the full wave function, which does not contain the outgoing two- and three-body scattered waves. For a better understanding of the three-body incident wave we consider first the two-body case.

\section{ASYMPTOTIC TWO-BODY SCATTERING WAVE FUNCTIONS}

We will be referring to the two-body Coulomb scattering throughout this work. Therefore we present here some important relations for the two-body scattering. Let us consider two charged particles with mass $m_{i}$ and charge $Z_{i} e, i=1,2$, interacting via the pure Coulomb potential $V=Z_{1} Z_{2} e^{2} / r$. The scattering of two particles is described by the Schrödinger equation

$$
\{E-H\} \psi_{\mathbf{k}}^{(+)}(\mathbf{r})=0 .
$$

$E=k^{2} /(2 \mu)$ is the relative kinetic energy of the interacting particles 1 and $2, H=-\Delta_{\mathbf{r}} /(2 \mu)+V$ is two-body Hamiltonian, and $\mu=m_{1} m_{2} /\left(m_{1}+m_{2}\right)$ is the reduced mass of particles 1 and 2. For the pure Coulomb interaction case Eq. (6) can be solved analytically. Substituting

$$
\psi_{\mathbf{k}}^{(+)}(\mathbf{r})=e^{i \mathbf{k} \cdot \mathbf{r}} N_{1} F_{1}(-i \eta, 1, i \zeta)
$$

where $\eta=Z_{1} Z_{2} e^{2} \mu / k$ is the Coulomb parameter, into Eq. (6) gives the differential equation for the confluent hypergeometric function (also called the Kummer function)

$$
\left[\frac{\Delta_{\mathbf{r}}}{2 \mu}+\frac{i \mathbf{k} \cdot \nabla_{\mathbf{r}}}{\mu}-V\right]{ }_{1} F_{1}(-i \eta, 1, i \zeta)=0,
$$

$N=e^{-\pi \eta / 2} \Gamma(1+i \eta)$ is the normalization factor, and parabolic coordinate $\zeta=k r-\mathbf{k} \cdot \mathbf{r} .{ }_{1} F_{1}(-i \eta, 1, i \zeta)$ is called the Kummer function because Eq. (8) rewritten in terms of $z=i \zeta$ becomes the Kummer differential equation $[8,9]$ :

$$
z \frac{d^{2}{ }_{1} F_{1}(a, c ; z)}{d z^{2}}+(c-z) \frac{d_{1} F_{1}(a, c ; z)}{d z}-a_{1} F_{1}(a, c ; z)=0 .
$$

Here $a=-i \eta, c=1$. Note that the Kummer function ${ }_{1} F_{1}(a, c ; z)$ is a regular solution at $\zeta=0($ or $r=0)$ of the Kummer equation. Correspondingly, $\psi_{\mathbf{k}}^{(+)}(\mathbf{r})$ given by Eq. (7) is the normalized regular solution of the two-body Coulomb scattering problem. The Kummer function can be expressed in terms of the Whittaker functions $W_{\lambda, \mu}(z)$ using Eqs. (9.220.3) and (9.233.2) of [10]: 


$$
\begin{aligned}
{ }_{1} F_{1}(-i \eta, 1 ; i \zeta)= & \frac{1}{\Gamma(1+i \eta)} e^{\pi \eta} \frac{1}{(i \zeta)^{1 / 2}} e^{i \zeta / 2} W_{1 / 2+i \eta, 0}(i \zeta) \\
& +\frac{1}{\Gamma(-i \eta)} e^{-i \pi(1 / 2+i \eta)} \frac{1}{(i \zeta)^{1 / 2}} e^{i \zeta / 2} \\
& \times W_{-1 / 2-i \eta, 0}\left(e^{-i \pi} i \zeta\right)
\end{aligned}
$$

Each term of Eq. (10) also satisfies the Kummer differential equation (9) providing a singular solution. Substituting Eq. (10) into the Kummer equation leads to the Whittaker differential equation for each term:

$$
\frac{d^{2} W_{\lambda, 0}(z)}{d z^{2}}+\left(-\frac{1}{4}+\frac{\lambda}{z}+\frac{\frac{1}{4}}{z^{2}}\right) W_{\lambda, 0}(z)=0 .
$$

Here $\lambda= \pm\left(\frac{1}{2}+i \eta\right)$ and $z= \pm i \zeta$. Evidently both Whittaker functions in Eq. (10) satisfy the same Whittaker equation because it is invariant under simultaneous transformation $z \rightarrow-z, \lambda \rightarrow-\lambda$. Coming back to the normalized regular solution of the Schrödinger equation we can present it as a sum of two singular solutions:

$$
\psi_{\mathbf{k}}^{(+)}(\mathbf{r})=e^{i \mathbf{k} \cdot \mathbf{r}} N_{1} F_{1}(-i \eta, 1, i \zeta)=\psi_{\mathbf{k}}^{(0)}(\mathbf{r})+\psi_{\mathbf{k}}^{(s c)}(\mathbf{r})
$$

The first singular solution, as we will see below, is the incident wave

$$
\psi_{\mathbf{k}}^{(0)}(\mathbf{r})=e^{i \mathbf{k} \cdot \mathbf{r}} \mathcal{F}^{(1)}(\zeta)
$$

and the second singular solution is the scattered wave

$$
\begin{gathered}
\psi_{\mathbf{k}}^{(s c)}(\mathbf{r})=e^{i \mathbf{k} \cdot \mathbf{r}} \mathcal{F}^{(2)}(\zeta) \\
\mathcal{F}^{(1)}(\zeta)=e^{\pi \eta / 2}(i \zeta)^{-1 / 2} e^{i \zeta / 2} W_{i \eta+\frac{1}{2}, 0}(i \zeta) \\
\mathcal{F}^{(2)}(\zeta)=-i \frac{\Gamma(1+i \eta)}{\Gamma(-i \eta)} e^{\pi \eta / 2}(i \zeta)^{-1 / 2} e^{i \zeta / 2} W_{-i \eta-\frac{1}{2}, 0}\left(e^{-i \pi} i \zeta\right)
\end{gathered}
$$

Evidently, for $\eta=0$ the incident wave becomes the plane wave $e^{i \mathbf{k} \cdot \mathbf{r}}$ and the scattered wave just disappears. It follows from Eq. (9.227) of [10] that $(|z|>0)$

$$
W_{1 / 2,0}(z)=e^{-z / 2} z^{1 / 2},
$$

and from Eq. (9.222.2) of [10]

$$
W_{-1 / 2,0}(z)=e^{-z / 2} z^{1 / 2} \int_{0}^{\infty} d t e^{-t} \frac{1}{t+z} .
$$

Taking into account the asymptotic behavior at $|z| \rightarrow \infty$ of the Whittaker function, Eq. (9.227) of [10],

$$
W_{\lambda, 0}(z)^{|z| \rightarrow \infty}=z^{\lambda} e^{-z / 2}\left[1-\frac{\left(\lambda-\frac{1}{2}\right)^{2}}{z}+O\left(\frac{1}{z^{2}}\right)\right],
$$

we derive the asymptotic behavior of $\mathcal{F}^{(1)}(\zeta)$ :

$$
\mathcal{F}^{(1)}(i \zeta)=e^{|\zeta| \rightarrow \ln \zeta}\left[1+O\left(\frac{1}{i \zeta}\right)\right] .
$$

Correspondingly the asymptotic behavior of the Coulomb distorted incident wave

$$
\psi_{\mathbf{k}}^{(0)}(\mathbf{r})=e^{i \mathbf{k} \cdot \mathbf{r}} e^{i \eta \ln \zeta}\left[1+O\left(\frac{1}{i \zeta}\right)\right] .
$$

The asymptotic behavior of $\mathcal{F}^{(2)}(\zeta)$ is given by

$$
\mathcal{F}^{(2)}(i \zeta)^{\zeta \rightarrow \infty}=f^{C} \frac{e^{i \zeta}}{r} e^{-i \eta \ln 2 k r}\left[1+O\left(\frac{1}{i \zeta}\right)\right],
$$

where $f^{C}$ is the on-the-energy-shell Coulomb scattering amplitude:

$$
f^{C}=-\eta \frac{\Gamma(1+i \eta)}{\Gamma(1-i \eta)}(-i)^{-i \eta} e^{\pi \eta / 2} \frac{e^{-i \eta \ln \sin ^{2}(\theta / 2)}}{2 k \sin ^{2} \frac{\theta}{2}} .
$$

The asymptotic behavior of the scattered wave is given by

$$
\psi_{\mathbf{k}}^{(s c)}(\mathbf{r}) \stackrel{r \rightarrow \infty}{=} f^{C} \frac{e^{i k r}}{r} e^{-i \eta \ln 2 k r}\left[1+O\left(\frac{1}{i \zeta}\right)\right] .
$$

Taking into account Eqs. (12), (21), and (24) we get the asymptotic behavior of the Coulomb scattering wave function for a system of two particles in the coordinate space:

$$
\begin{aligned}
\psi_{\mathbf{k}}^{(+)}(\mathbf{r})= & e^{i \mathbf{k} \cdot \mathbf{r}} e^{i \eta \ln \zeta}\left[1+O\left(\frac{1}{i \zeta}\right)\right] \\
& +f^{C} \frac{e^{i k r}}{r} e^{-i \eta \ln 2 k r}\left[1+O\left(\frac{1}{i \zeta}\right)\right] .
\end{aligned}
$$

Note that this asymptotic behavior is valid only for $|\zeta| \rightarrow \infty$. For $r \rightarrow \infty$ it is valid for all directions in the configuration space except for the so-called singular direction, for which $\hat{\mathbf{k}} \cdot \hat{\mathbf{r}}=1$. Here $\hat{\mathbf{a}}=\mathbf{a} / a$.

One can observe a very interesting feature in the case of the two-body Coulomb scattering. The regular solution, according to Eq. (12), consists of two singular solutions, incident and scattered wave, and each of them also satisfies the Schrödinger equation. Correspondingly, the asymptotic Coulomb scattering wave function consists of two terms. The first one, $e^{i \mathbf{k} \cdot \mathbf{r}} e^{i \eta \ln \zeta}[1+O(1 / i \zeta)]$ is the asymptotic form of $e^{i \mathbf{k} \cdot \mathbf{r}} \mathcal{F}^{(1)}(i \zeta)$ and represents the Coulomb distorted incident wave. The Coulomb distortion not only generates a logarithmic phase factor $\eta \ln \zeta$ as an additional phase factor to the plane wave phase factor $\mathbf{k} \cdot \mathbf{r}$, but it also generates an infinite series in powers of $1 / \zeta$. This is in contrast to the two-body scattering problem for particles interacting via short-range potentials, where the incident wave is given just by the plane wave. The second term in Eq. (25) is the asymptotic form for $e^{i \mathbf{k} \cdot \mathbf{r}} \mathcal{F}^{(2)}(i \zeta)$. It generates the outgoing two-body spherical wave times the Coulomb scattering amplitude and also contains a factor that can be written as an asymptotic expansion in powers of $1 / \zeta$. 


\section{ASYMPTOTIC THREE-BODY INCIDENT WAVE}

After the consideration of the incident wave for the twobody case, it is easier to proceed to the incident wave for the three-body case. By definition, the incident three-body wave is the part of the total three-body scattering wave function of the first type, which does not contain two- and three-body scattered waves. We have shown that the two-body incident wave is a singular solution of the two-body Schrödinger equation. It is natural to ask whether the three-body incident wave is a solution of the three-body Schrödinger equation. An educated guess tells us that the answer may be "yes." First, the scattering wave function of the first type, which consists of the three-body incident and scattered waves (twoand three-body), is a regular solution of the three-body Schrödinger equation. However, there are also singular solutions of the three-body Schrödinger equation. And it is very plausible that the three-body incident wave is one of the singular solutions while the scattered wave represents another singular solution. However, we cannot prove it until an analytical expression for the three-body incident wave is available.

Our goal in this work is to derive the asymptotic incident three-body wave function in the leading orders $O(1)$, $O\left(1 / \rho_{\alpha}\right), O\left(1 / \rho_{\alpha}^{2}\right)$ in the asymptotic region $\Omega_{\alpha}$, where particles $\beta$ and $\gamma$ are close to each other and far away from particle $\alpha$. We will demonstrate that the terms of order $O\left(1 / \rho_{\alpha}^{2}\right)$ can be derived without explicit solution of the three-body Schrödinger equation. In principle, the method we use can be applied to get even the higher order terms of the three-body incident wave but it is worth mentioning that the next order term in the asymptotic expansion of the threebody incident wave $O\left(1 / \rho_{\alpha}^{3}\right)$ is inferior to the outgoing threebody scattered wave $O\left(1 / R^{5 / 2}\right)$. Hence for practical applications one needs to know the three-body scattered wave before getting the term $O\left(1 / \rho_{\alpha}^{3}\right)$ in the asymptotic expansion of the three-body incident wave. A knowledge of the threebody incident wave up to terms $O\left(1 / \rho_{\alpha}^{3}\right)$ allows us to write down the leading asymptotic terms of the three-body scattering wave function of the first type in the asymptotic region $\Omega_{\alpha}$ up to terms $O\left(1 / \rho_{\alpha}^{3}\right)$. Note that the expressions for the asymptotic incident three-body wave in two other asymptotic regions $\Omega_{\beta}$ and $\Omega_{\gamma}$ can be derived by the simple cyclic permutation of indexes $\alpha, \beta$, and $\gamma$. As we have mentioned earlier, the asymptotic incident three-body wave is the part of the total three-body scattering wave function of the first type, which does not contain two- and three-body scattered waves. This wave function should smoothly transform into the asymptotic incident three-body wave function in the asymptotic region $\Omega_{0}$. This smooth matching is an important part of the boundary conditions that provides a unique solution.

The leading asymptotic term of the three-body incident wave in $\Omega_{0}$ derived by Redmond $[11,12]$ is given by

$$
\Psi_{\mathbf{k}_{\alpha}, \mathbf{q}_{\alpha}}^{(0)(+)}\left(\mathbf{r}_{\alpha}, \boldsymbol{\rho}_{\alpha}\right)=e^{i \mathbf{k}_{\alpha} \cdot \mathbf{r}_{\alpha}} e^{i \mathbf{q}_{\alpha} \cdot \boldsymbol{\rho}_{\alpha}} \prod_{\nu=\alpha, \beta, \gamma} e^{i \eta_{\nu} \ln \zeta_{\nu}}
$$

where

$$
\begin{gathered}
\zeta_{\nu}=k_{\nu} r_{\nu}-\mathbf{k}_{\nu} \cdot \mathbf{r}_{\nu} \\
\eta_{\alpha}=\frac{Z_{\beta} Z_{\gamma} e^{2} \mu_{\alpha}}{k_{\alpha}}
\end{gathered}
$$

is the Coulomb parameter of particles $\beta$ and $\gamma, \mu_{\alpha}$ is the reduced mass of particles $\beta$ and $\gamma$. It is the three-body Coulomb distorted plane wave. For practical applications Merkuriev [1] and Garibotti and Miraglia [13] extended the asymptotic Redmond term by substituting the confluent hypergeometric functions for the exponential Coulomb distortion factors. This extended wave function, often called the $3 \mathrm{C}$ wave function, is given by

$$
\Psi_{\mathbf{k}_{\alpha}, \mathbf{q}_{\alpha}}^{(3 C)(+)}\left(\mathbf{r}_{\alpha}, \boldsymbol{\rho}_{\alpha}\right)=e^{i \mathbf{k}_{\alpha} \cdot \mathbf{r}_{\alpha}} e^{i \mathbf{q}_{\alpha} \cdot \boldsymbol{\rho}_{\alpha}} \prod_{\nu=\alpha, \beta, \gamma} F_{\nu}\left(\zeta_{\nu}\right),
$$

where

$$
F_{\nu}\left(\zeta_{\nu}\right)=N_{\nu 1} F_{1}\left(-i \eta_{\nu}, 1 ; i \zeta_{\nu}\right),
$$

${ }_{1} F_{1}\left(-i \eta_{\nu}, 1 ; i \zeta_{\nu}\right)$ is the confluent hypergeometric function and

$$
N_{\nu}=e^{-\pi \eta_{\nu} / 2} \Gamma\left(1+i \eta_{\nu}\right)
$$

Note that

$$
\psi_{\mathbf{k}_{\alpha}}\left(\mathbf{r}_{\alpha}\right)=e^{i \mathbf{k}_{\alpha} \cdot \mathbf{r}_{\alpha} F_{\alpha}\left(\zeta_{\alpha}\right)}
$$

is the Coulomb scattering wave function of particles $\beta$ and $\gamma$ moving with the relative momentum $\mathbf{k}_{\alpha}$ and is wellbehaved even in the singular directions $\left(\zeta_{\alpha}<C\right.$ for $\left.r_{\alpha} \rightarrow \infty\right)$ where Redmond's asymptotic term is not determined. If any of the particles is neutral, then the resulting asymptotic solution becomes the plane wave for the neutral particle and the exact two-body scattering wave function for the charged pair. However, neither Redmond's asymptotic term $\Psi_{\mathbf{k}_{\alpha}, \mathbf{q}_{\alpha}}^{(0)(+)}\left(\mathbf{r}_{\alpha}, \boldsymbol{\rho}_{\alpha}\right)$ nor the $3 \mathrm{C}$ wave function $\Psi_{\mathbf{k}_{\alpha}, \mathbf{q}_{\alpha}}^{(3 C)(+)}\left(\mathbf{r}_{\alpha}, \boldsymbol{\rho}_{\alpha}\right)$ are asymptotic solutions of the Schrödinger equation in the asymptotic domains $\Omega_{\nu}, \nu=\alpha, \beta, \gamma$. Redmond's asymptotic term, by construction, satisfies the asymptotic Schrödinger equation up to terms $O\left(1 / r_{\alpha}^{2}, 1 / r_{\beta}^{2}, 1 / r_{\gamma}^{2}\right)$. However, in the asymptotic region, $\Omega_{\nu}$, the distance between the particles of pair $\nu$ is limited: $r_{\nu}<C^{\prime}$. Hence the terms $O\left(1 / r_{\nu}\right)$ are not small and the potential $V_{\nu}^{C}$ in the Schrödinger equation has to be compensated exactly rather than asymptotically as happens when we use Redmond's asymptotic wave function in $\Omega_{0}$. In the $3 \mathrm{C}$ wave function two very important effects are absent. Consider, for example, the asymptotic region $\Omega_{\alpha}$. In this region $r_{\alpha} \ll \rho_{\alpha}$. Hence the two-body relative motion of particles $\beta$ and $\gamma$ is distorted by the Coulomb field of the third particle $\alpha$ [2]. The second evident defect in the $3 \mathrm{C}$ function is the absence of the nuclear interaction between particles $\beta$ and $\gamma$, which can be close enough to each other in $\Omega_{\alpha}$. Nevertheless, the $3 \mathrm{C}$ wave function can be used as a starting point to derive leading asymptotic terms of the threebody incident wave in $\Omega_{\alpha}[2,3]$, because this asymptotic three-body incident wave should match Redmond's asymptotic term in $\Omega_{0}$. We will demonstrate now how important the condition of the matching of the asymptotic wave functions is on the border of different asymptotic regions [2]. 
In Redmond's asymptotic incident wave three logarithmic phase factors appear, one phase factor for each pair rather than two phase factors in the factorized solution. It is a very important conclusion. In all the conventional approaches for breakup processes, including coupled channels codes like FRESCO, the three-body scattering wave function is approximated by the factorized one. From the consideration above, it is clear that if Coulomb interactions are important, such an approximation is not accurate. If the interactions are shortrange, the factorized solution matches the asymptotic solution in $\Omega_{0}$ and is justified in the asymptotic region $\Omega_{\alpha}$. It has been shown in $[2,3]$ that the actual asymptotic solution of the asymptotic Schrödinger equation $\Psi_{\mathbf{k}_{\alpha} \mathbf{q}_{\alpha}}^{(a s)}\left(\mathbf{r}_{\alpha}, \boldsymbol{\rho}_{\alpha}\right)$, which matches Redmond's asymptotic term in $\Omega_{0}$, cannot be written in a factorized form and has a quite complicated behavior. In $[2,3]$ all the leading asymptotic terms up to $O\left(1 / \rho_{\alpha}^{2}\right)$ of the asymptotic wave function $\Psi_{\mathbf{k}_{\alpha} \mathbf{q}_{\alpha}}^{(a s)}\left(\mathbf{r}_{\alpha}, \boldsymbol{\rho}_{\alpha}\right)$ have been derived in the asymptotic region $\Omega_{\alpha}^{\alpha}$. In this work we will present a derivation of the expansion of the asymptotic wave function, $\Psi_{\mathbf{k}_{\alpha} \mathbf{q}_{\alpha}}^{(a s)}\left(\mathbf{r}_{\alpha}, \boldsymbol{\rho}_{\alpha}\right)$, up to terms $O\left(1 / \rho_{\alpha}^{3}\right)$. The derived asymptotic expansion contains all the terms $O(1), O\left(1 / \rho_{\alpha}\right)$, and $O\left(1 / \rho_{\alpha}^{2}\right)$. Since we are looking for the terms $O\left(1 / \rho_{\alpha}^{2}\right)$, we need to keep the terms up to $O\left(1 / \rho_{\alpha}^{3}\right)$. Instead of the asymptotic expansion of the Coulomb potentials $V_{\beta}^{C}\left(\mathbf{r}_{\beta}\right)$ and $V_{\gamma}^{C}\left(\mathbf{r}_{\gamma}\right)$ in terms of $1 / \rho_{\alpha}$, we will start our derivation from the exact three-body Schrödinger equation (3). The terms of $O\left(1 / \rho_{\alpha}^{3}\right)$ will be dropped later. The asymptotic wave function in $\Omega_{\alpha}$ should match the asymptotic wave function in $\Omega_{0}$. The $3 \mathrm{C}$ wave function satisfies Eq. (3) up to terms $O\left(1 / r_{\alpha}^{2}, 1 / \rho_{\alpha}^{2}\right)$ and we can use it as the initial wave function. However, this wave function should be modified to satisfy the Schrödinger equation in $\Omega_{\alpha}$. Note that usually in the literature it is assumed that Redmond's asymptotic term satisfies the Schrödinger equation in $\Omega_{0}$ in the leading order only. First we will show that the $3 \mathrm{C}$ wave function satisfies the Schrödinger equation in $\Omega_{0}$ up terms of order $O\left(1 / r_{\nu}^{2}\right)$. To this end we just substitute the $3 \mathrm{C}$ wave function (29) into the Schrödinger equation (3):

$$
\begin{aligned}
(E- & \left.T_{\mathbf{r}_{\alpha}}-T_{\boldsymbol{\rho}_{\alpha}}-V\right)\left[e^{i \mathbf{k}_{\alpha} \cdot \mathbf{r}_{\alpha}+i \mathbf{q}_{\alpha} \cdot \boldsymbol{\rho}_{\alpha}} \varphi_{\mathbf{k}_{\alpha}}\left(\mathbf{r}_{\alpha}\right) \varphi_{\mathbf{k}_{\beta}}\left(\mathbf{r}_{\beta}\right) \varphi_{\mathbf{k}_{\gamma}}\left(\mathbf{r}_{\gamma}\right)\right] \\
= & e^{i \mathbf{k}_{\alpha} \cdot \mathbf{r}_{\alpha}+i \mathbf{q}_{\alpha} \cdot \boldsymbol{\rho}_{\alpha}} \varphi_{\mathbf{k}_{\beta}}\left(\mathbf{r}_{\beta}\right) \varphi_{\mathbf{k}_{\gamma}}\left(\mathbf{r}_{\gamma}\right)\left[\frac{\Delta_{\mathbf{r}_{\alpha}}}{2 \mu_{\alpha}}+\frac{i \mathbf{k}_{\alpha} \cdot \nabla_{\mathbf{r}_{\alpha}}}{\mu_{\alpha}}-V_{\alpha}+\frac{\nabla_{\mathbf{r}_{\alpha}} \varphi_{\mathbf{k}_{\gamma}} \cdot \nabla_{\mathbf{r}_{\alpha}} \varphi_{\mathbf{k}_{\beta}}}{\mu_{\alpha} \varphi_{\mathbf{k}_{\beta}} \varphi_{\mathbf{k}_{\gamma}}}+\frac{\boldsymbol{\nabla}_{\boldsymbol{\rho}_{\alpha}} \varphi_{\mathbf{k}_{\gamma}} \cdot \boldsymbol{\nabla}_{\boldsymbol{\rho}_{\alpha}} \varphi_{\mathbf{k}_{\beta}}}{M_{\alpha} \varphi_{\mathbf{k}_{\beta}} \varphi_{\mathbf{k}_{\gamma}}}\right] \varphi_{\mathbf{k}_{\alpha}}\left(\mathbf{r}_{\alpha}\right) \\
& +e^{i \mathbf{k}_{\beta} \cdot \mathbf{r}_{\beta}+i \mathbf{q}_{\beta} \cdot \boldsymbol{\rho}_{\beta}} \varphi_{\mathbf{k}_{\alpha}}\left(\mathbf{r}_{\alpha}\right) \varphi_{\mathbf{k}_{\gamma}}\left(\mathbf{r}_{\gamma}\right)\left[\frac{\Delta_{\mathbf{r}_{\beta}}}{2 \mu_{\beta}}+\frac{i \mathbf{k}_{\beta} \cdot \nabla_{\mathbf{r}_{\beta}}}{\mu_{\beta}}-V_{\beta}+\frac{\boldsymbol{\nabla}_{\mathbf{r}_{\beta}} \varphi_{\mathbf{k}_{\gamma}} \cdot \nabla_{\mathbf{r}_{\beta}} \varphi_{k_{\alpha}}}{\mu_{\beta} \varphi_{\mathbf{k}_{\alpha}} \varphi_{\mathbf{k}_{\gamma}}}+\frac{\boldsymbol{\nabla}_{\boldsymbol{\rho}_{\beta}} \varphi_{\mathbf{k}_{\gamma}} \cdot \nabla_{\boldsymbol{\rho}_{\beta}} \varphi_{\mathbf{k}_{\alpha}}}{M_{\beta} \varphi_{\mathbf{k}_{\alpha}} \varphi_{\mathbf{k}_{\gamma}}}\right] \varphi_{\mathbf{k}_{\beta}}\left(\mathbf{r}_{\beta}\right) \\
& +e^{i \mathbf{k}_{\gamma} \cdot \mathbf{r}_{\gamma}+i \mathbf{q}_{\gamma} \cdot \boldsymbol{\rho}_{\gamma} \varphi_{\mathbf{k}_{\alpha}}\left(\mathbf{r}_{\alpha}\right) \varphi_{\mathbf{k}_{\beta}}\left(\mathbf{r}_{\beta}\right)}\left[\frac{\Delta_{\mathbf{r}_{\gamma}}}{2 \mu_{\gamma}}+\frac{i \mathbf{k}_{\gamma} \cdot \nabla_{\mathbf{r}_{\gamma}}}{\mu_{\gamma}}-V_{\gamma}+\frac{\nabla_{\mathbf{r}_{\gamma}} \varphi_{\mathbf{k}_{\beta}} \cdot \nabla_{\mathbf{r}_{\gamma}} \varphi_{k_{\alpha}}}{\mu_{\gamma} \varphi_{\mathbf{k}_{\alpha}} \varphi_{\mathbf{k}_{\beta}}}+\frac{\nabla_{\boldsymbol{\rho}_{\gamma}} \varphi_{\mathbf{k}_{\beta}} \cdot \nabla_{\boldsymbol{\rho}_{\gamma}} \varphi_{\mathbf{k}_{\alpha}}}{M_{\gamma} \varphi_{\mathbf{k}_{\alpha}} \varphi_{\mathbf{k}_{\beta}}}\right] \varphi_{\mathbf{k}_{\gamma}}\left(\mathbf{r}_{\gamma}\right) .
\end{aligned}
$$

Here

$$
\varphi_{\mathbf{k}_{\nu}}\left(\mathbf{r}_{\nu}\right)=N_{\nu} F\left(-i \eta_{\nu}, 1 ; i \zeta_{\nu}\right)=\mathcal{F}_{\nu}^{(1)}\left(i \zeta_{\nu}\right)+\mathcal{F}_{\nu}^{(2)}\left(i \zeta_{\nu}\right) .
$$

Taking into account

$$
\left[\frac{\Delta_{\mathbf{r}_{v}}}{2 \mu_{\nu}}+\frac{i \mathbf{k}_{\nu} \cdot \nabla_{\mathbf{r}_{v}}}{\mu_{\nu}}-V_{\nu}\right] \varphi_{\mathbf{k}_{\nu}}\left(\mathbf{r}_{\nu}\right)=0
$$

we derive

$$
\begin{aligned}
(E & \left.-T_{\mathbf{r}_{\alpha}}-T_{\boldsymbol{\rho}_{\alpha}}-V\right)\left[e^{i \mathbf{k}_{\alpha} \cdot \mathbf{r}_{\alpha}+i \mathbf{q}_{\alpha} \cdot \boldsymbol{\rho}_{\alpha}} \varphi_{\mathbf{k}_{\alpha}}\left(\mathbf{r}_{\alpha}\right) \varphi_{\mathbf{k}_{\beta}}\left(\mathbf{r}_{\beta}\right) \varphi_{\mathbf{k}_{\gamma}}\left(\mathbf{r}_{\gamma}\right)\right] \\
& =O\left(1 / r_{\alpha}^{2}, 1 / r_{\beta}^{2}, 1 / r_{\gamma}^{2}\right) .
\end{aligned}
$$

We did not use any approximation to get Eq. (36). Thus the $3 \mathrm{C}$ wave function indeed satisfies the Schrödinger equation in $\Omega_{0}$ up to the terms $O\left(1 / r_{\alpha}^{2}, 1 / r_{\beta}^{2}, 1 / r_{\gamma}^{2}\right)$, i.e., after substitution of the $3 \mathrm{C}$ wave function into the Schrödinger equation all the terms of order $O(1)$ and $O\left(1 / r_{\alpha}\right)$ are exactly compensated. Hence the $3 \mathrm{C}$ wave function can be used as a starting wave function with proper modifications to look for an asymptotic solution in $\Omega_{\alpha}$. Taking into account Eq. (34) we can rewrite the $3 \mathrm{C}$ wave function in a form which is suitable for consideration in the $\Omega_{\alpha}$ asymptotic domain:

$$
\begin{aligned}
& \Psi_{\mathbf{k}_{\alpha}, \mathbf{q}_{\alpha}}^{(3 C)(+)}\left(\mathbf{r}_{\alpha}, \boldsymbol{\rho}_{\alpha}\right)=e^{i \mathbf{k}_{\alpha} \cdot \mathbf{r}_{\alpha}} e^{i \mathbf{q}_{\alpha} \cdot \boldsymbol{\rho}_{\alpha}} \\
& \times\left[\mathcal{F}_{\beta}^{(1)}\left(i \zeta_{\beta}\right) \mathcal{F}_{\gamma}^{(1)}\left(i \zeta_{\gamma}\right) N_{\alpha} F_{\alpha}\left(i \zeta_{\alpha}\right)\right. \\
& +\mathcal{F}_{\beta}^{(2)}\left(i \zeta_{\beta}\right) \mathcal{F}_{\gamma}^{(1)}\left(i \zeta_{\gamma}\right) N_{\alpha} F_{\alpha}\left(i \zeta_{\alpha}\right) \\
& +\mathcal{F}_{\beta}^{(1)}\left(i \zeta_{\beta}\right) \mathcal{F}_{\gamma}^{(2)}\left(i \zeta_{\gamma}\right) N_{\alpha} F_{\alpha}\left(i \zeta_{\alpha}\right) \\
& \left.+\mathcal{F}_{\beta}^{(2)}\left(i \zeta_{\beta}\right) \mathcal{F}_{\gamma}^{(2)}\left(i \zeta_{\gamma}\right) N_{\alpha} F_{\alpha}\left(i \zeta_{\alpha}\right)\right] \text {. }
\end{aligned}
$$

Here, asymptotically, for $\left|\zeta_{\nu}\right| \rightarrow \infty$, the first term $\mathcal{F}^{(1)}\left(\zeta_{\nu}\right)$ $\sim O(1)$ and the second term $\mathcal{F}^{(2)}\left(\zeta_{\nu}\right) \sim O\left(1 / \zeta_{\nu}\right)$. Hence in the asymptotic domain $\Omega_{\alpha} \mathcal{F}^{(1)}\left(\zeta_{\nu}\right)$ and $\mathcal{F}^{(2)}\left(\zeta_{\nu}\right), \nu=\beta, \gamma$, can be treated asymptotically while $\varphi_{\mathbf{k}_{\alpha}}\left(\mathbf{r}_{\alpha}\right)$ should be considered explicitly, because $\Omega_{\alpha}$ includes the region, where $r_{\alpha}$ is 
limited. Moreover, in the asymptotic region $\Omega_{\alpha}$ the relative motion of particles $\beta$ and $\gamma$ is distorted by the third particle $\alpha$ due to the long-range Coulomb interaction. It means that the wave function of the relative motion of particles $\beta$ and $\gamma$ in $\Omega_{\alpha}$ will be different from the wave function $e^{i \mathbf{k} \mathbf{k}^{\prime} \cdot \mathbf{r}} \alpha_{N} N_{\alpha} F_{\alpha}\left(i \zeta_{\alpha}\right)$ describing the relative motion of particles $\beta$ and $\gamma$ in the absence of the third particle. Since interacting particles $\beta$ and $\gamma$ can be close to each other in $\Omega_{\alpha}$, their nuclear interaction should also be taken into account. Following [3] we replace each $N_{\alpha} F_{\alpha}\left(i \zeta_{\alpha}\right)$ in Eq. (38) by the corresponding unknown function $\varphi_{\alpha}^{(n m)}\left(\mathbf{r}_{\alpha}\right), n, m=1,2$ :

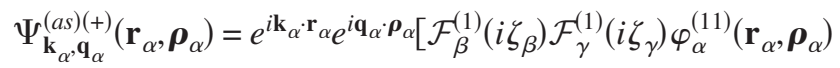

$$
\begin{aligned}
& +\mathcal{F}_{\beta}^{(2)}\left(i \zeta_{\beta}\right) \mathcal{F}_{\gamma}^{(1)}\left(i \zeta_{\gamma}\right) \varphi_{\alpha}^{(21)}\left(\mathbf{r}_{\alpha}, \boldsymbol{\rho}_{\alpha}\right) \\
& +\mathcal{F}_{\beta}^{(1)}\left(i \zeta_{\beta}\right) \mathcal{F}_{\gamma}^{(2)}\left(i \zeta_{\gamma}\right) \varphi_{\alpha}^{(12)}\left(\mathbf{r}_{\alpha}, \boldsymbol{\rho}_{\alpha}\right) \\
& \left.+\mathcal{F}_{\beta}^{(2)}\left(i \zeta_{\beta}\right) \mathcal{F}_{\gamma}^{(2)}\left(i \zeta_{\gamma}\right) \varphi_{\alpha}^{(22)}\left(\mathbf{r}_{\alpha}, \boldsymbol{\rho}_{\alpha}\right)\right]
\end{aligned}
$$

Derivation of $\varphi_{\alpha}^{(n m)}\left(\mathbf{r}_{\alpha}\right), n, m=1,2$ is our final goal. Now we substitute Eq. (38) into the Schrödinger equation (3). When substituting Eq. (38) into the Schrödinger equation we assume that each term of the sum (38) satisfies the Schrödinger equation. Moreover, as we will see, each function $\varphi_{\alpha}\left(\mathbf{r}_{\alpha}, \boldsymbol{\rho}_{\alpha}\right)$ depends on the preceding functions $\mathcal{F}_{\beta}^{(n)}\left(i \zeta_{\beta}\right) \mathcal{F}_{\gamma}^{(m)}\left(i \zeta_{\gamma}\right)$ where $n, m=1,2$, i.e., for each term in (38) the modification is different. We also take into account that

$$
\left(\frac{1}{2 \mu_{\nu}} \boldsymbol{\Delta}_{\mathbf{r}_{\nu}}+i \frac{1}{\mu_{\nu}} \mathbf{k}_{\nu} \cdot \boldsymbol{\nabla}_{\mathbf{r}_{\nu}}-V_{\nu}^{C}\right) \mathcal{F}_{\nu}^{(1,2)}\left(i \zeta_{\nu}\right)=0 .
$$

Substitution of the first term of Eq. (38) into the Schrödinger equation generates the equation for $\varphi_{\alpha}^{(11)}\left(\mathbf{r}_{\alpha}\right)$ :

$$
\begin{aligned}
& \mathcal{F}_{\beta}^{(1)}\left(i \zeta_{\beta}\right) \mathcal{F}_{\gamma}^{(1)}\left(i \zeta_{\gamma}\right)\left[\frac{1}{2 \mu_{\alpha}} \boldsymbol{\Delta}_{\mathbf{r}_{\alpha}}+\frac{1}{2 M_{\alpha}} \boldsymbol{\Delta}_{\boldsymbol{\rho}_{\alpha}}+i \frac{1}{\mu_{\alpha}} \mathbf{k}_{\alpha} \cdot \nabla_{\mathbf{r}_{\alpha}}\right. \\
& +i \frac{1}{M_{\alpha}} \mathbf{q}_{\alpha} \cdot \nabla_{\boldsymbol{\rho}_{\alpha}}+\frac{1}{\mu_{\alpha}} \sum_{\nu=\beta, \gamma} \boldsymbol{\nabla}_{\mathbf{r}_{\alpha}} \ln \mathcal{F}_{\nu}^{(1)}\left(i \zeta_{\nu}\right) \cdot \nabla_{\mathbf{r}_{\alpha}} \\
& \quad+\frac{1}{M_{\alpha}} \sum_{\nu=\beta, \gamma} \boldsymbol{\nabla}_{\boldsymbol{\rho}_{\alpha}} \ln \mathcal{F}_{\nu}^{(1)}\left(i \zeta_{\nu}\right) \cdot \nabla_{\boldsymbol{\rho}_{\alpha}}-V_{\alpha}\left(\mathbf{r}_{\alpha}\right) \\
& \quad+\frac{1}{\mu_{\alpha}} \boldsymbol{\nabla}_{\mathbf{r}_{\alpha}} \ln \mathcal{F}_{\beta}^{(1)}\left(i \zeta_{\beta}\right) \cdot \nabla_{\mathbf{r}_{\alpha}} \ln \mathcal{F}_{\gamma}^{(1)}\left(i \zeta_{\gamma}\right) \\
& \left.\quad+\frac{1}{M_{\alpha}} \nabla_{\boldsymbol{\rho}_{\alpha}} \ln \mathcal{F}_{\beta}^{(1)}\left(i \zeta_{\beta}\right) \cdot \nabla_{\boldsymbol{\rho}_{\alpha}} \ln \mathcal{F}_{\gamma}^{(1)}\left(i \zeta_{\gamma}\right)\right] \varphi_{\alpha}^{(11)}\left(\mathbf{r}_{\alpha}, \boldsymbol{\rho}_{\alpha}\right) \\
& =0 .
\end{aligned}
$$

Since particles $\beta$ and $\gamma$ are allowed to be close in $\Omega_{\alpha}$ their interaction potential is given by the sum of the Coulomb and nuclear potentials. Now we will simplify this equation by dropping all the terms $O\left(1 / \rho_{\alpha}^{3}\right)$ and explicitly compensate all the terms $O(1), O\left(1 / \rho_{\alpha}\right), O\left(1 / \rho_{\alpha}^{2}\right)$. We consider only the nonsingular directions, i.e., $\hat{\mathbf{k}}_{\nu} \cdot \hat{\mathbf{r}}_{\nu} \neq 1, \nu=\beta, \gamma$. To analyze the fifth term in the brackets we use equations

$$
\begin{aligned}
& \mathcal{F}_{\nu}^{(1)}\left(i \zeta_{\nu}\right)=\tilde{\mathcal{F}}_{\nu}^{(1)}\left(i \zeta_{\nu}\right)\left[1-i \frac{\eta_{\nu}^{2}}{\zeta_{\nu}}+O\left(1 / \zeta_{\nu}^{2}\right)\right] \\
& \tilde{\mathcal{F}}_{\nu}^{(1)}\left(i \zeta_{\nu}\right)=e^{i \eta_{\nu} \ln \zeta_{\nu}} \\
& \boldsymbol{\nabla}_{\mathbf{r}_{\alpha}} \ln \mathcal{F}_{\nu}^{(1)}\left(i \zeta_{\nu}\right)=\nabla_{\mathbf{r}_{\alpha}} \ln \widetilde{\mathcal{F}}_{\nu}^{(1)}\left(i \zeta_{\nu}\right)-i \frac{m_{\nu}}{m_{\beta \gamma}} \frac{\eta_{\nu}^{2}}{k_{\nu} r_{\nu}^{2}} \frac{\hat{\mathbf{r}}_{\nu}-\hat{\mathbf{k}}_{\nu}}{\left(1-\hat{\mathbf{k}}_{\nu} \cdot \hat{\mathbf{r}}_{\nu}\right)^{2}} \\
& +O\left(1 / r_{v}^{3}\right) \\
& \boldsymbol{\nabla}_{\mathbf{r}_{\alpha}} \ln \widetilde{\mathcal{F}}_{\nu}^{(1)}\left(i \zeta_{\nu}\right)=\nabla_{\mathbf{r}_{\alpha}} e^{m_{\nu} / m_{\beta \gamma} \epsilon_{\nu \alpha} \mathbf{r}_{\alpha} \cdot \boldsymbol{\nabla}_{\alpha}} \ln \tilde{\mathcal{F}}_{\nu}^{(1)}\left(i \zeta_{\nu \alpha}\right) \\
& =\epsilon_{\nu \alpha} \frac{m_{\nu}}{m_{\beta \gamma}} \nabla_{\boldsymbol{\rho}_{\alpha}} \ln \widetilde{\mathcal{F}}_{\nu}^{(1)}\left(i \zeta_{\nu \alpha}\right) \\
& +\frac{m_{\nu}^{2}}{m_{\beta \gamma}^{2}}\left(\mathbf{r}_{\alpha} \cdot \boldsymbol{\nabla}_{\boldsymbol{\rho}_{\alpha}}\right) \boldsymbol{\nabla}_{\boldsymbol{\rho}_{\alpha}} \ln \tilde{\mathcal{F}}_{\nu}^{(1)}\left(i \zeta_{\nu \alpha}\right), \\
& \boldsymbol{\nabla}_{\boldsymbol{\rho}_{\alpha}} \ln \widetilde{\mathcal{F}}_{\nu}^{(1)}\left(i \zeta_{\nu \alpha}\right)=i \eta_{\nu} \boldsymbol{\epsilon}_{\nu \alpha} \frac{1}{\rho_{\alpha}} \frac{\hat{\mathbf{k}}_{\nu}-\boldsymbol{\epsilon}_{\alpha \nu} \dot{\hat{\boldsymbol{\rho}}}_{\alpha}}{1-\boldsymbol{\epsilon}_{\alpha \nu} \hat{\mathbf{k}}_{\nu} \cdot \hat{\boldsymbol{\rho}}_{\alpha}}+O\left(\frac{1}{\rho_{\alpha}^{2}}\right), \\
& \boldsymbol{\nabla}_{\mathbf{r}_{\alpha}}\left[-i \frac{\eta_{\nu}^{2}}{\zeta_{\nu}}\right]=i \eta_{\nu}^{2} \frac{m_{\nu}}{m_{\beta \gamma}} \frac{1}{k_{\nu} \rho_{\alpha}^{2}} \frac{\hat{\mathbf{k}}_{\nu}-\boldsymbol{\epsilon}_{\alpha \nu} \hat{\boldsymbol{\rho}}_{\alpha}}{\left(1-\boldsymbol{\epsilon}_{\alpha \nu} \hat{\mathbf{k}}_{\nu} \cdot \hat{\boldsymbol{\rho}}_{\alpha}\right)^{2}}+O\left(\frac{1}{\rho_{\alpha}^{3}}\right) .
\end{aligned}
$$

Here, $\zeta_{\nu \alpha}=k_{\nu} \rho_{\alpha}-\epsilon_{\alpha \nu} \mathbf{k}_{\nu} \cdot \boldsymbol{\rho}_{\alpha}$. To estimate the sixth and the ninth terms we use equations

$$
\begin{aligned}
\boldsymbol{\nabla}_{\boldsymbol{\rho}_{\alpha}} \ln \mathcal{F}_{\nu}^{(1)}(i) & =i \eta_{\nu} \frac{1}{r_{\nu}} \epsilon_{\nu \alpha} \frac{\hat{\mathbf{k}}_{\nu}-\hat{\mathbf{r}}_{\nu}}{1-\hat{\mathbf{k}}_{\nu} \cdot \hat{\mathbf{r}}_{\nu}}+O\left(\frac{1}{r_{\nu}^{2}}\right) \\
& =i \eta_{\nu} \epsilon_{\nu \alpha} \frac{1}{\rho_{\alpha}} \frac{\hat{\mathbf{k}}_{\nu}-\boldsymbol{\epsilon}_{\alpha \nu} \hat{\boldsymbol{\rho}}_{\alpha}}{1-\boldsymbol{\epsilon}_{\alpha \nu} \hat{\mathbf{k}}_{\nu} \cdot \hat{\boldsymbol{\rho}}_{\alpha}}+O\left(\frac{1}{\rho_{\alpha}^{2}}\right) .
\end{aligned}
$$

To estimate the eighth term we use equation

$$
\nabla_{\mathbf{r}_{\alpha}} \ln \mathcal{F}_{\nu}^{(1)}\left(i \zeta_{\nu}\right)=i \eta_{\nu} \frac{m_{\nu}}{m_{\beta \gamma}} \frac{1}{r_{\nu}} \frac{\hat{\mathbf{k}}_{\nu}-\hat{\mathbf{r}}_{\nu}}{1-\hat{\mathbf{k}}_{\nu} \cdot \hat{\mathbf{r}}_{\nu}} .
$$

Note that in the $\Omega_{\alpha}$ radius $r_{\alpha}$ is limited a priori (more strictly, it is allowed to grow but slower than $\rho_{\alpha}$ ). That is why we cannot use an asymptotic expansion in terms of $1 / \zeta_{\alpha}$ in the asymptotic region $\Omega_{\alpha}$. Equations (44)-(46) and (48) are valid only in $\Omega_{\alpha}$, while Eqs. (43), (47), and (49) are valid both in $\Omega_{0}$ and $\Omega_{\alpha}$.

Thus we reduced a three-body problem in the asymptotic domain $\Omega_{\alpha}$ to a two-body problem: we need to find a solution of Eq. (40), which describes the relative motion of particles $\beta$ and $\gamma$ in the presence of the third particle $\alpha$, which is 
far away, but it still distorts the relative motion of particles $\beta$ and $\gamma$ due to the long-range Coulomb interaction. This distortion results in the dependence of $\varphi_{\alpha}^{(11)}\left(\mathbf{r}_{\alpha}, \boldsymbol{\rho}_{\alpha}\right)$ on $\boldsymbol{\rho}_{\alpha}$. When $\rho_{\alpha}$ increases, this distortion should be weakened. Hence, $\varphi_{\alpha}^{(11)}\left(\mathbf{r}_{\alpha}, \boldsymbol{\rho}_{\alpha}\right)$ actually depends on $1 / \rho_{\alpha}$ and

$$
\boldsymbol{\nabla}_{\boldsymbol{\rho}_{\alpha}} \varphi_{\alpha}^{(11)}\left(\mathbf{r}_{\alpha}, \boldsymbol{\rho}_{\alpha}\right) \sim \frac{1}{\rho_{\alpha}^{2}}
$$

Because of that we may drop the second and sixth terms in Eq. (40) and rewrite it in the form

$$
\begin{aligned}
& {\left[\frac{1}{2 \mu_{\alpha}} \boldsymbol{\Delta}_{\mathbf{r}_{\alpha}}+i \frac{1}{\mu_{\alpha}} \mathbf{k}_{\alpha} \cdot \nabla_{\mathbf{r}_{\alpha}}+i \frac{1}{M_{\alpha}} \mathbf{q}_{\alpha} \cdot \nabla_{\boldsymbol{\rho}_{\alpha}}+\frac{1}{\mu_{\alpha}} \sum_{\nu=\beta, \gamma} \boldsymbol{\nabla}_{\mathbf{r}_{\alpha}} \ln \mathcal{F}_{\nu}^{(1)}\left(i \zeta_{\nu}\right) \cdot \nabla_{\mathbf{r}_{\alpha}}-V_{\alpha}\left(\mathbf{r}_{\alpha}\right)\right.} \\
& \left.\quad+\frac{1}{\mu_{\alpha}} \nabla_{\mathbf{r}_{\alpha}} \ln \mathcal{F}_{\beta}^{(1)}\left(i \zeta_{\beta}\right) \cdot \nabla_{\mathbf{r}_{\alpha}} \ln \mathcal{F}_{\gamma}^{(1)}\left(i \zeta_{\gamma}\right)+\frac{1}{M_{\alpha}} \nabla_{\boldsymbol{\rho}_{\alpha}} \ln \mathcal{F}_{\beta}^{(1)}\left(i \zeta_{\beta}\right) \cdot \nabla_{\boldsymbol{\rho}_{\alpha}} \ln \mathcal{F}_{\gamma}^{(1)}\left(i \zeta_{\gamma}\right)\right] \varphi_{\alpha}^{(11)}\left(\mathbf{r}_{\alpha}, \boldsymbol{\rho}_{\alpha}\right)=0 .
\end{aligned}
$$

The last two terms are of $O\left(1 / \rho_{\alpha}^{2}\right)$. Note that to satisfy this equation up to terms of $O\left(1 / \rho_{\alpha}^{3}\right)$ all the terms of $O\left(1 / \rho_{\alpha}^{2}\right)$ must be compensated. Taking into account Eqs. (43), (44), and (46) we can rewrite Eq. (51) as

$$
\begin{aligned}
& {\left[\frac{1}{2 \mu_{\alpha}} \boldsymbol{\Delta}_{\mathbf{r}_{\alpha}}+i \frac{1}{\mu_{\alpha}} \mathbf{k}_{\alpha}^{(11)}\left(\boldsymbol{\rho}_{\alpha}\right) \cdot \nabla_{\mathbf{r}_{\alpha}}+i \frac{1}{M_{\alpha}} \mathbf{q}_{\alpha} \cdot \nabla_{\boldsymbol{\rho}_{\alpha}}+\frac{1}{\mu_{\alpha}} \sum_{\nu=\beta, \gamma} \frac{m_{\nu}^{2}}{m_{\beta \gamma}^{2}}\left(\mathbf{r}_{\alpha} \cdot \nabla_{\boldsymbol{\rho}_{\alpha}}\right)\left(\boldsymbol{\nabla}_{\boldsymbol{\rho}_{\alpha}} \ln \mathcal{F}_{\nu}^{(1)}\left(i \zeta_{\nu \alpha}\right) \cdot \nabla_{\mathbf{r}_{\alpha}}\right)-V_{\alpha}\left(\mathbf{r}_{\alpha}\right)\right.} \\
& \left.\quad+\left(\boldsymbol{\epsilon}_{\beta \alpha} \boldsymbol{\epsilon}_{\gamma \alpha} \frac{1}{m_{\beta \gamma}}+\frac{1}{M_{\alpha}}\right) \boldsymbol{\nabla}_{\boldsymbol{\rho}_{\alpha}} \ln \mathcal{F}_{\beta}^{(1)}\left(i \zeta_{\beta \alpha}\right) \cdot \nabla_{\boldsymbol{\rho}_{\alpha}} \ln \mathcal{F}_{\gamma}^{(1)}\left(i \zeta_{\gamma \alpha}\right)\right] \boldsymbol{\varphi}_{\alpha}^{(11)}\left(\mathbf{r}_{\alpha}, \boldsymbol{\rho}_{\alpha}\right)=O\left(1 / \rho_{\alpha}^{3}\right) .
\end{aligned}
$$

We introduced here a new local momentum

$$
\begin{aligned}
\mathbf{k}_{\alpha}^{(11)}= & \mathbf{k}_{\alpha}-i \sum_{\nu=\beta, \gamma} \frac{m_{\nu}}{m_{\beta \gamma}} \\
& \times\left[\boldsymbol{\epsilon}_{\nu \alpha} \boldsymbol{\nabla}_{\boldsymbol{\rho}_{\alpha}} \ln \tilde{\mathcal{F}}_{\nu}^{(1)}\left(i \zeta_{\nu \alpha}\right)+i \eta_{\nu}^{2} \frac{1}{k_{\nu} \rho_{\alpha}^{2}} \frac{\hat{\mathbf{k}}_{\nu}-\boldsymbol{\epsilon}_{\alpha \nu} \hat{\boldsymbol{\rho}}_{\alpha}}{\left(1-\boldsymbol{\epsilon}_{\alpha \nu} \hat{\mathbf{k}}_{\nu} \cdot \hat{\boldsymbol{\rho}}_{\alpha}\right)^{2}}\right] .
\end{aligned}
$$

Note that variables $\boldsymbol{\nabla}_{\mathbf{r}_{\alpha}}$ and $\boldsymbol{\nabla}_{\boldsymbol{\rho}_{\alpha}}$ are mixed up only in the fourth term of Eq. (52). We are looking for a solution in the form

$$
\varphi_{\alpha}^{(11)}\left(\mathbf{r}_{\alpha}, \boldsymbol{\rho}_{\alpha}\right)=\varphi_{\alpha(0)}^{(11)}\left(\mathbf{r}_{\alpha}, \boldsymbol{\rho}_{\alpha}\right)\left(1+\frac{\chi\left(\hat{\boldsymbol{\rho}}_{\alpha}\right)}{\rho_{\alpha}}\right)+\frac{\varphi_{\alpha(1)}^{(11)}\left(\mathbf{r}_{\alpha}, \boldsymbol{\rho}_{\alpha}\right)}{\rho_{\alpha}^{2}},
$$

where $\varphi_{\alpha(0)}^{(11)}\left(\mathbf{r}_{\alpha}, \boldsymbol{\rho}_{\alpha}\right)$ is a solution of

$$
\left[\frac{1}{2 \mu_{\alpha}} \boldsymbol{\Delta}_{\mathbf{r}_{\alpha}}+i \frac{1}{\mu_{\alpha}} \mathbf{k}_{\alpha}^{(11)}\left(\boldsymbol{\rho}_{\alpha}\right) \cdot \nabla_{\mathbf{r}_{\alpha}}-V_{\alpha}\left(\mathbf{r}_{\alpha}\right)\right] \varphi_{\alpha(0)}^{(11)}\left(\mathbf{r}_{\alpha}, \boldsymbol{\rho}_{\alpha}\right)=0
$$

$\chi\left(\hat{\boldsymbol{\rho}}_{\alpha}\right) \sim O(1)$ and is a solution of the first order differential equation

$$
\begin{aligned}
i \frac{1}{M_{\alpha}} \mathbf{q}_{\alpha} \cdot \nabla_{\boldsymbol{\rho}_{\alpha}} \frac{\chi\left(\hat{\boldsymbol{\rho}}_{\alpha}\right)}{\rho_{\alpha}}= & -\left(\boldsymbol{\epsilon}_{\beta \alpha} \epsilon_{\gamma \alpha} \frac{1}{m_{\beta \gamma}}+\frac{1}{M_{\alpha}}\right) \boldsymbol{\nabla}_{\boldsymbol{\rho}_{\alpha}} \\
& \times \ln \tilde{\mathcal{F}}_{\beta}^{(1)}\left(i \zeta_{\beta \alpha}\right) \cdot \nabla_{\boldsymbol{\rho}_{\alpha}} \ln \tilde{\mathcal{F}}_{\gamma}^{(1)}\left(i \zeta_{\gamma \alpha}\right) .
\end{aligned}
$$

Here, $\boldsymbol{\epsilon}_{\beta \alpha} \boldsymbol{\epsilon}_{\gamma \alpha}\left(1 / m_{\beta \gamma}\right)+1 / M_{\alpha}=1 / m_{\alpha}$. Finally $\varphi_{\alpha(1)}^{(11)}\left(\mathbf{r}_{\alpha}, \boldsymbol{\rho}_{\alpha}\right)$ $\sim O(1)$ is a solution of the inhomogeneous equation

$$
\begin{aligned}
& {\left[\frac{1}{2 \mu_{\alpha}} \boldsymbol{\Delta}_{\mathbf{r}_{\alpha}}+i \frac{1}{\mu_{\alpha}} \mathbf{k}_{\alpha} \cdot \nabla_{\mathbf{r}_{\alpha}}-V_{\alpha}\left(\mathbf{r}_{\alpha}\right)\right] \varphi_{\alpha(1)}^{(11)}\left(\mathbf{r}_{\alpha}, \boldsymbol{\rho}_{\alpha}\right)} \\
& =-i \frac{\rho_{\alpha}^{2}}{M_{\alpha}} \mathbf{q}_{\alpha} \cdot \nabla_{\boldsymbol{\rho}_{\alpha}} \varphi_{\alpha(0)}^{(11)}\left(\mathbf{r}_{\alpha}\right)-\frac{\rho_{\alpha}^{2}}{\mu_{\alpha}} \sum_{\nu=\beta, \gamma} \frac{m_{\nu}^{2}}{m_{\beta \gamma}^{2}}\left(\mathbf{r}_{\alpha} \cdot \nabla_{\boldsymbol{\rho}_{\alpha}}\right) \\
& \quad \times \nabla_{\boldsymbol{\rho}_{\alpha}} \ln \tilde{\mathcal{F}}_{\nu}^{(1)}\left(i \zeta_{\nu \alpha}\right) \cdot \nabla_{\mathbf{r}_{\alpha}} \varphi_{\alpha(0)}^{(11)}\left(\mathbf{r}_{\alpha}, \boldsymbol{\rho}_{\alpha}\right) .
\end{aligned}
$$

Note that all the equations (55)-(57) are "two-body" differential equations. On the left-hand side they contain gradients and Laplacians over only one of the variables, $\mathbf{r}_{\alpha}$ or $\boldsymbol{\rho}_{\alpha}$. Therefore these equations can be solved numerically.

Now we consider the second term of Eq. (38). It satisfies the equation 


$$
\begin{aligned}
& \mathcal{F}_{\beta}^{(2)}\left(i \zeta_{\beta}\right) \mathcal{F}_{\gamma}^{(1)}\left(i \zeta_{\gamma}\right)\left[\frac{1}{2 \mu_{\alpha}} \boldsymbol{\Delta}_{\mathbf{r}_{\alpha}}+\frac{1}{2 M_{\alpha}} \boldsymbol{\Delta}_{\boldsymbol{\rho}_{\alpha}}+i \frac{1}{\mu_{\alpha}} \mathbf{k}_{\alpha} \cdot \boldsymbol{\nabla}_{\mathbf{r}_{\alpha}}+i \frac{1}{M_{\alpha}} \mathbf{q}_{\alpha} \cdot \nabla_{\boldsymbol{\rho}_{\alpha}}+\frac{1}{\mu_{\alpha}}\left[\boldsymbol{\nabla}_{\mathbf{r}_{\alpha}} \ln \mathcal{F}_{\beta}^{(2)}\left(i \zeta_{\beta}\right)+\nabla_{\mathbf{r}_{\alpha}} \ln \mathcal{F}_{\gamma}^{(1)}\left(i \zeta_{\gamma}\right)\right] \cdot \nabla_{\mathbf{r}_{\alpha}}\right. \\
& +\frac{1}{M_{\alpha}}\left[\boldsymbol{\nabla}_{\boldsymbol{\rho}_{\alpha}} \ln \mathcal{F}_{\beta}^{(2)}\left(i \zeta_{\beta}\right)+\boldsymbol{\nabla}_{\boldsymbol{\rho}_{\alpha}} \ln \mathcal{F}_{\gamma}^{(1)}\left(i \zeta_{\gamma}\right)\right] \cdot \boldsymbol{\nabla}_{\boldsymbol{\rho}_{\alpha}}-V_{\alpha}\left(\mathbf{r}_{\alpha}\right)+\frac{1}{\mu_{\alpha}} \boldsymbol{\nabla}_{\mathbf{r}_{\alpha}} \ln \mathcal{F}_{\beta}^{(2)}\left(i \zeta_{\beta}\right) \cdot \nabla_{\mathbf{r}_{\alpha}} \ln \mathcal{F}_{\gamma}^{(1)}\left(i \zeta_{\gamma}\right) \\
& \left.+\frac{1}{M_{\alpha}} \boldsymbol{\nabla}_{\boldsymbol{\rho}_{\alpha}} \ln \mathcal{F}_{\beta}^{(2)}\left(i \zeta_{\beta}\right) \cdot \boldsymbol{\nabla}_{\boldsymbol{\rho}_{\alpha}} \ln \mathcal{F}_{\gamma}^{(1)}\left(i \zeta_{\gamma}\right)\right] \varphi_{\alpha}^{(21)}\left(\mathbf{r}_{\alpha}, \boldsymbol{\rho}_{\alpha}\right)=O\left(1 / \rho_{\alpha}^{3}\right) .
\end{aligned}
$$

Here, in the nonsingular directions $\left(\hat{\mathbf{k}}_{\nu} \cdot \hat{\mathbf{r}}_{\nu} \neq 1, \nu \neq \alpha\right)$

$$
\mathcal{F}_{\nu}^{(2)}\left(i \zeta_{\nu}\right)^{\zeta_{\nu} \rightarrow \infty}=\eta_{\nu} \frac{\Gamma\left(1+i \eta_{\nu}\right)}{\Gamma\left(1-i \eta_{\nu}\right)} \frac{e^{-i \eta_{\nu} \ln \zeta_{\nu}}}{\zeta_{\nu}} e^{i \zeta_{\nu}}\left[1+O\left(\frac{1}{\zeta_{\nu}}\right)\right] \text {. }
$$

Also, in the nonsingular directions for $\nu \neq \alpha$

$$
\begin{aligned}
\boldsymbol{\nabla}_{\mathbf{r}_{\alpha}} \ln \mathcal{F}_{\nu}^{(2)}\left(i \zeta_{\nu}\right) & =i \nabla_{\mathbf{r}_{\alpha} \zeta_{\nu}}+O\left(1 / r_{\nu}\right) \\
& =i \frac{m_{\nu}}{m_{\beta \gamma}} k_{\nu}\left(\hat{\mathbf{k}}_{\nu}-\hat{\mathbf{r}}_{\nu}\right)+O\left(1 / r_{\nu}\right), \\
& =i \frac{m_{\nu}}{m_{\beta \gamma}} k_{\nu}\left(\hat{\mathbf{k}}_{\nu}-\epsilon_{\alpha \nu} \hat{\boldsymbol{\rho}}_{\alpha}\right)+O\left(1 / \rho_{\alpha}\right),
\end{aligned}
$$

and

$$
\begin{aligned}
\boldsymbol{\nabla}_{\boldsymbol{\rho}_{\alpha}} \ln \mathcal{F}_{\nu}^{(2)}\left(i \zeta_{\nu}\right) & =i \boldsymbol{\nabla}_{\boldsymbol{\rho}_{\alpha} \zeta_{\nu}}+O\left(1 / r_{\nu}\right) \\
& =i \epsilon_{\nu \alpha}\left(-k_{\nu} \hat{\mathbf{r}}_{\nu}+\mathbf{k}_{\nu}\right)+O\left(1 / r_{\nu}\right) \\
& =i k_{\nu}\left(\hat{\boldsymbol{\rho}}_{\alpha}-\epsilon_{\alpha \nu} \hat{\mathbf{k}}_{\nu}\right)+O\left(1 / \rho_{\alpha}\right)
\end{aligned}
$$

When deriving (58) we took into account that

$$
\left(\frac{1}{2 \mu_{\nu}} \boldsymbol{\Delta}_{\mathbf{r}_{\nu}}+i \frac{1}{\mu_{\nu}} \mathbf{k}_{\nu} \cdot \nabla_{\mathbf{r}_{\nu}}-V_{\nu}^{C}\right) \mathcal{F}_{\nu}^{(2)}\left(i \zeta_{\nu}\right)=0
$$

To get an asymptotic equation from Eq. (58) which is valid up to $O\left(1 / \rho_{\alpha}^{3}\right)$, all the coefficients of $O(1), O\left(1 / \rho_{\alpha}\right)$, and $O\left(1 / \rho_{\alpha}^{2}\right)$ should be kept in the left-hand side of the equation. Since in the nonsingular directions in the $\Omega_{\alpha}$ region, $\mathcal{F}_{\beta}^{(2)}\left(i \zeta_{\beta}\right) \sim O\left(1 / \rho_{\alpha}\right)$ only coefficients of $O(1)$ and $O\left(1 / \rho_{\alpha}\right)$ in the brackets of Eq. (58) should be left. Taking into account Eqs. (43), (44), (61), and (63) we get

$$
\begin{aligned}
& {\left[\frac{1}{2 \mu_{\alpha}} \boldsymbol{\Delta}_{\mathbf{r}_{\alpha}}+i \frac{1}{\mu_{\alpha}} \mathbf{k}_{\alpha}^{(21)}\left(\boldsymbol{\rho}_{\alpha}\right) \cdot \nabla_{\mathbf{r}_{\alpha}}-V_{\alpha}\left(\mathbf{r}_{\alpha}\right)\right.} \\
& \quad+i \frac{1}{\mu_{\alpha}} \frac{m_{\beta}^{2}}{m_{\beta \gamma}^{2}} k_{\beta} \frac{1}{\rho_{\alpha}}\left[\mathbf{r}_{\alpha}-\hat{\boldsymbol{\rho}}_{\alpha}\left(\hat{\boldsymbol{\rho}}_{\alpha} \cdot \mathbf{r}_{\alpha}\right)\right] \cdot \nabla_{\mathbf{r}_{\alpha}} \\
& \quad+i \frac{1}{M_{\alpha}} \mathbf{q}_{\alpha}^{(21)} \cdot \nabla_{\boldsymbol{\rho}_{\alpha}}-i \epsilon_{\alpha \beta} \frac{1}{m_{\alpha}} k_{\beta}\left(\hat{\mathbf{k}}_{\beta}-\boldsymbol{\epsilon}_{\alpha \beta} \hat{\boldsymbol{\rho}}_{\alpha}\right) \cdot \nabla_{\boldsymbol{\rho}_{\alpha}} \\
& \left.\quad \times \ln \tilde{\mathcal{F}}_{\gamma}^{(1)}\left(i \zeta_{\gamma \alpha}\right)\right] \varphi_{\alpha}^{(21)}\left(\mathbf{r}_{\alpha}, \boldsymbol{\rho}_{\alpha}\right)=O\left(1 / \rho_{\alpha}^{2}\right) .
\end{aligned}
$$

Here $\boldsymbol{\nabla}_{\boldsymbol{\rho}_{\alpha}} \ln \mathcal{F}_{\gamma}^{(1)}\left(i \zeta_{\gamma \alpha}\right)$ is given by Eq. (45). We also introduced new local momenta

$$
\begin{aligned}
\mathbf{k}_{\alpha}^{(21)}\left(\boldsymbol{\rho}_{\alpha}\right)= & \mathbf{k}_{\alpha}+\frac{m_{\beta}}{m_{\beta \gamma}} k_{\beta}\left(\hat{\mathbf{k}}_{\beta}-\boldsymbol{\epsilon}_{\alpha \beta} \hat{\boldsymbol{\rho}}_{\alpha}\right) \\
& +i\left(i \eta_{\beta}+1\right) \frac{m_{\beta}}{m_{\beta \gamma}} \frac{1}{\boldsymbol{\rho}_{\alpha}} \frac{\hat{\mathbf{k}}_{\beta}-\boldsymbol{\epsilon}_{\alpha \beta} \hat{\boldsymbol{\rho}}_{\alpha}}{1-\boldsymbol{\epsilon}_{\alpha \beta} \hat{\mathbf{k}}_{\beta} \cdot \hat{\boldsymbol{\rho}}_{\alpha}} \\
& +\eta_{\gamma} \frac{m_{\gamma}}{m_{\beta \gamma}} \frac{1}{\boldsymbol{\rho}_{\alpha}} \frac{\hat{\mathbf{k}}_{\gamma}-\boldsymbol{\epsilon}_{\alpha \gamma} \hat{\boldsymbol{\rho}}_{\alpha}}{1-\boldsymbol{\epsilon}_{\alpha \gamma} \hat{\mathbf{k}}_{\gamma} \cdot \hat{\boldsymbol{\rho}}_{\alpha}},
\end{aligned}
$$

and

$$
\mathbf{q}_{\alpha}^{(21)}\left(\boldsymbol{\rho}_{\alpha}\right)=\mathbf{q}_{\alpha}+k_{\beta}\left(\hat{\boldsymbol{\rho}}_{\alpha}-\epsilon_{\alpha \beta} \hat{\mathbf{k}}_{\beta}\right) .
$$

We also took into account that for $\nu \neq \sigma \neq \tau, \quad \nu \neq \tau$, $\epsilon_{\nu \tau} \epsilon_{\nu \sigma}=-1$, and

$$
\begin{aligned}
& -\boldsymbol{\epsilon}_{\alpha \gamma} \frac{1}{m_{\beta \gamma}}\left(\hat{\mathbf{k}}_{\beta}-\boldsymbol{\epsilon}_{\alpha \beta} \hat{\boldsymbol{\rho}}_{\alpha}\right)+\frac{1}{M_{\alpha}}\left(\boldsymbol{\rho}_{\alpha}-\boldsymbol{\epsilon}_{\alpha \beta} \hat{\mathbf{k}}_{\beta}\right) \\
& =-\boldsymbol{\epsilon}_{\alpha \beta} \frac{1}{m_{\alpha}}\left(\hat{\mathbf{k}}_{\beta}-\boldsymbol{\epsilon}_{\alpha \beta} \hat{\boldsymbol{\rho}}_{\alpha}\right) .
\end{aligned}
$$

We are looking for a solution of Eq. (66) in the form

$$
\varphi_{\alpha}^{(21)}\left(\mathbf{r}_{\alpha}, \boldsymbol{\rho}_{\alpha}\right)=\varphi_{\alpha(0)}^{(21)}\left(\mathbf{r}_{\alpha}, \boldsymbol{\rho}_{\alpha}\right)+\frac{\varphi_{\alpha(1)}^{(21)}\left(\mathbf{r}_{\alpha}, \boldsymbol{\rho}_{\alpha}\right)}{\rho_{\alpha}},
$$

where $\varphi_{\alpha(0)}^{(21)}\left(\mathbf{r}_{\alpha}, \boldsymbol{\rho}_{\alpha}\right)$ satisfies

$$
\left[\frac{1}{2 \mu_{\alpha}} \boldsymbol{\Delta}_{\mathbf{r}_{\alpha}}+i \frac{1}{\mu_{\alpha}} \mathbf{k}_{\alpha}^{(21)}\left(\boldsymbol{\rho}_{\alpha}\right) \cdot \nabla_{\mathbf{r}_{\alpha}}-V_{\alpha}\left(\mathbf{r}_{\alpha}\right)\right] \varphi_{\alpha(0)}^{(21)}\left(\mathbf{r}_{\alpha}, \boldsymbol{\rho}_{\alpha}\right)=0 .
$$

Finally $\varphi_{\alpha(1)}^{(21)}\left(\mathbf{r}_{\alpha}, \boldsymbol{\rho}_{\alpha}\right) \sim O(1)$ is a solution of the equation

$$
\begin{gathered}
{\left[\frac{1}{2 \mu_{\alpha}} \boldsymbol{\Delta}_{\mathbf{r}_{\alpha}}+i \frac{1}{\mu_{\alpha}} \mathbf{k}_{\alpha}^{(21)}\left(\boldsymbol{\rho}_{\alpha}\right) \cdot \nabla_{\mathbf{r}_{\alpha}}-V_{\alpha}\left(\mathbf{r}_{\alpha}\right)\right] \varphi_{\alpha(1)}^{(21)}\left(\mathbf{r}_{\alpha}, \boldsymbol{\rho}_{\alpha}\right)} \\
=-\left[i \frac{1}{\mu_{\alpha}} \frac{m_{\beta}^{2}}{m_{\beta \gamma}^{2}} k_{\beta}\left[\mathbf{r}_{\alpha}-\hat{\boldsymbol{\rho}}_{\alpha}\left(\hat{\boldsymbol{\rho}}_{\alpha} \cdot \mathbf{r}_{\alpha}\right)\right] \cdot \nabla_{\mathbf{r}_{\alpha}}\right] \varphi_{\alpha(0)}^{(21)}\left(\mathbf{r}_{\alpha}, \boldsymbol{\rho}_{\alpha}\right) \\
-i \frac{\rho_{\alpha}}{M_{\alpha}} \mathbf{q}_{\alpha}^{(21)} \cdot \nabla_{\boldsymbol{\rho}_{\alpha}} \varphi_{\alpha(0)}^{(21)}\left(\mathbf{r}_{\alpha}, \boldsymbol{\rho}_{\alpha}\right)+i \epsilon_{\alpha \beta} \frac{\rho_{\alpha}}{m_{\alpha}} k_{\beta}\left(\hat{\mathbf{k}}_{\beta}\right. \\
\left.-\boldsymbol{\epsilon}_{\alpha \beta} \hat{\boldsymbol{\rho}}_{\alpha}\right) \cdot \nabla_{\boldsymbol{\rho}_{\alpha}} \ln \widetilde{\mathcal{F}}_{\gamma}^{(1)}\left(i \zeta_{\gamma \alpha}\right) \varphi_{\alpha(0)}^{(21)}\left(\mathbf{r}_{\alpha}, \boldsymbol{\rho}_{\alpha}\right) .
\end{gathered}
$$


Since in Eq. (71) we keep only the terms of order $O\left(1 / \rho_{\alpha}\right)$, local momentum $\mathbf{k}_{\alpha}^{(21)}\left(\boldsymbol{\rho}_{\alpha}\right)$ can be replaced by

$$
\mathbf{k}_{\alpha(0)}^{(21)}\left(\boldsymbol{\rho}_{\alpha}\right)=\mathbf{k}_{\alpha}+\frac{m_{\beta}}{m_{\beta \gamma}} k_{\beta}\left(\hat{\mathbf{k}}_{\beta}-\epsilon_{\alpha \beta} \hat{\boldsymbol{\rho}}_{\alpha}\right)
$$

A formal solution of Eq. (71) is

$$
\begin{aligned}
& \varphi_{\alpha(1)}^{(21)}\left(\mathbf{r}_{\alpha}, \boldsymbol{\rho}_{\alpha}\right)=\varphi_{\alpha(0)}^{(21)}\left(\mathbf{r}_{\alpha}, \boldsymbol{\rho}_{\alpha}\right)+e^{-\mathbf{k}_{\alpha}^{(21)}\left(\boldsymbol{\rho}_{\alpha}\right) \cdot \mathbf{r}_{\alpha}} \int d \mathbf{r}_{\alpha}^{\prime} G\left(\mathbf{r}_{\alpha}, \mathbf{r}_{\alpha}^{\prime}\right) e^{\mathbf{k}_{\alpha}^{(21)}\left(\boldsymbol{\rho}_{\alpha}\right) \cdot \mathbf{r}_{\alpha}^{\prime}}\left[-\left(i \frac{1}{\mu_{\alpha}} \frac{m_{\beta}^{2}}{m_{\beta \gamma}^{2}} k_{\beta}\left[\mathbf{r}_{\alpha}^{\prime}-\hat{\boldsymbol{\rho}}_{\alpha}\left(\hat{\boldsymbol{\rho}}_{\alpha} \cdot \mathbf{r}_{\alpha}^{\prime}\right)\right] \cdot \nabla_{\mathbf{r}_{\alpha}}\right) \varphi_{\alpha(0)}^{(21)}\left(\mathbf{r}_{\alpha}^{\prime}, \boldsymbol{\rho}_{\alpha}\right)\right. \\
& \left.-i \frac{1}{M_{\alpha}} \mathbf{q}_{\alpha}^{(21)}\left(\boldsymbol{\rho}_{\alpha}\right) \cdot \nabla_{\boldsymbol{\rho}_{\alpha}} \varphi_{\alpha(0)}^{(21)}\left(\mathbf{r}_{\alpha}^{\prime}, \boldsymbol{\rho}_{\alpha}\right)-i \epsilon_{\alpha \beta} \frac{1}{m_{\alpha}} k_{\beta}\left(\hat{\mathbf{k}}_{\beta}-\boldsymbol{\epsilon}_{\alpha \beta} \hat{\boldsymbol{\rho}}_{\alpha}\right) \cdot \nabla_{\boldsymbol{\rho}_{\alpha}} \ln \tilde{\mathcal{F}}_{\gamma}^{(1)}\left(i \zeta_{\gamma \alpha}^{\prime}\right) \varphi_{\alpha(0)}^{(21)}\left(\mathbf{r}_{\alpha}^{\prime}, \boldsymbol{\rho}_{\alpha}\right)\right] .
\end{aligned}
$$

Here $\varphi_{\alpha(0)}^{(21)}\left(\mathbf{r}_{\alpha}, \boldsymbol{\rho}_{\alpha}\right)$ is a solution of the homogeneous Eq. (38).

The third equation for $\varphi_{\alpha}^{(12)}\left(\mathbf{r}_{\alpha}, \boldsymbol{\rho}_{\alpha}\right)$ is obtained by substituting the third term in (38) to (3). Following the same steps, which we used to derive the second equation, or just interchanging $\beta \leftrightarrow \gamma$ in (58) we find $\varphi_{\alpha}^{(12)}\left(\mathbf{r}_{\alpha}, \boldsymbol{\rho}_{\alpha}\right)$ in the following form:

$$
\varphi_{\alpha}^{(12)}\left(\mathbf{r}_{\alpha}, \boldsymbol{\rho}_{\alpha}\right)=\varphi_{\alpha(0)}^{(12)}\left(\mathbf{r}_{\alpha}, \boldsymbol{\rho}_{\alpha}\right)+\frac{\varphi_{\alpha(1)}^{(12)}\left(\mathbf{r}_{\alpha}, \boldsymbol{\rho}_{\alpha}\right)}{\rho_{\alpha}},
$$

where $\varphi_{\alpha(0)}^{(12)}\left(\mathbf{r}_{\alpha}, \boldsymbol{\rho}_{\alpha}\right)$ is a solution of

$$
\left[\frac{1}{2 \mu_{\alpha}} \boldsymbol{\Delta}_{\mathbf{r}_{\alpha}}+i \frac{1}{\mu_{\alpha}} \mathbf{k}_{\alpha}^{(12)}\left(\boldsymbol{\rho}_{\alpha}\right) \cdot \nabla_{\mathbf{r}_{\alpha}}-V_{\alpha}\left(\mathbf{r}_{\alpha}\right)\right] \varphi_{\alpha(0)}^{(12)}\left(\mathbf{r}_{\alpha}, \boldsymbol{\rho}_{\alpha}\right)=0 .
$$

We can derive a similar equation to Eq. (71) for $\varphi_{\alpha(1)}^{(12)}\left(\mathbf{r}_{\alpha}, \boldsymbol{\rho}_{\alpha}\right)$ which has a formal solution

$$
\begin{aligned}
\varphi_{\alpha(1)}^{(12)}\left(\mathbf{r}_{\alpha}, \boldsymbol{\rho}_{\alpha}\right)= & \varphi_{\alpha(0)}^{(12)}\left(\mathbf{r}_{\alpha}, \boldsymbol{\rho}_{\alpha}\right)+e^{-\mathbf{k}_{\alpha}^{(12)}\left(\boldsymbol{\rho}_{\alpha}\right) \cdot \mathbf{r}_{\alpha} \int} \int d \mathbf{r}_{\alpha}^{\prime} G\left(\mathbf{r}_{\alpha}, \mathbf{r}_{\alpha}^{\prime}\right) e^{\mathbf{k}_{\alpha}^{(12)}\left(\boldsymbol{\rho}_{\alpha}\right) \cdot \mathbf{r}_{\alpha}^{\prime}}\left[-\left(i \frac{1}{\mu_{\alpha}} \frac{m_{\beta}^{2}}{m_{\beta \gamma}^{2}} k_{\beta}\left[\mathbf{r}_{\alpha}^{\prime}-\hat{\boldsymbol{\rho}}_{\alpha}\left(\hat{\boldsymbol{\rho}}_{\alpha} \cdot \mathbf{r}_{\alpha}^{\prime}\right)\right] \cdot \nabla_{\mathbf{r}_{\alpha}}\right) \varphi_{\alpha(0)}^{(12)}\left(\mathbf{r}_{\alpha}^{\prime}, \boldsymbol{\rho}_{\alpha}\right)\right. \\
& \left.-i \frac{1}{M_{\alpha}} \mathbf{q}_{\alpha}^{(12)}\left(\boldsymbol{\rho}_{\alpha}\right) \cdot \nabla_{\boldsymbol{\rho}_{\alpha}} \varphi_{\alpha(0)}^{(12)}\left(\mathbf{r}_{\alpha}^{\prime}, \boldsymbol{\rho}_{\alpha}\right)-i \epsilon_{\alpha \beta} \frac{1}{m_{\alpha}} k_{\beta}\left(\hat{\mathbf{k}}_{\beta}-\boldsymbol{\epsilon}_{\alpha \beta} \hat{\boldsymbol{\rho}}_{\alpha}\right) \cdot \boldsymbol{\nabla}_{\boldsymbol{\rho}_{\alpha}} \ln \tilde{\mathcal{F}}_{\gamma}^{(1)}\left(i \zeta_{\gamma \alpha}^{\prime}\right) \varphi_{\alpha(0)}^{(12)}\left(\mathbf{r}_{\alpha}^{\prime}, \boldsymbol{\rho}_{\alpha}\right)\right]
\end{aligned}
$$

The fourth equation can be derived after substituting the last term of Eq. (38) into Eq. (3) and it is automatically satisfied up to the terms of order $O\left(1 / \rho_{\alpha}^{3}\right)$ in $\Omega_{\alpha}$ because the product $\mathcal{F}_{\beta}^{(2)}\left(i \zeta_{\beta}\right) \mathcal{F}_{\gamma}^{(1)}\left(i \zeta_{\gamma}\right)=O\left(1 / \rho_{\alpha}^{2}\right)$. The fourth term in Eq. (38) leads to an equation for $\varphi_{\alpha}^{(22)}\left(\mathbf{r}_{\alpha}, \boldsymbol{\rho}_{\alpha}\right)$ :

$$
\begin{aligned}
& \mathcal{F}_{\beta}^{(2)}\left(i \zeta_{\beta}\right) \mathcal{F}_{\gamma}^{(2)}\left(i \zeta_{\gamma}\right)\left[\frac{1}{2 \mu_{\alpha}} \boldsymbol{\Delta}_{\mathbf{r}_{\alpha}}+\frac{1}{2 M_{\alpha}} \boldsymbol{\Delta}_{\boldsymbol{\rho}_{\alpha}}+i \frac{1}{\mu_{\alpha}} \mathbf{k}_{\alpha} \cdot \boldsymbol{\nabla}_{\mathbf{r}_{\alpha}}+i \frac{1}{M_{\alpha}} \mathbf{q}_{\alpha} \cdot \boldsymbol{\nabla}_{\boldsymbol{\rho}_{\alpha}}+\frac{1}{\mu_{\alpha}}\left[\boldsymbol{\nabla}_{\mathbf{r}_{\alpha}} \ln \mathcal{F}_{\beta}^{(2)}\left(i \zeta_{\beta}\right)+\nabla_{\mathbf{r}_{\alpha}} \ln \mathcal{F}_{\gamma}^{(2)}\left(i \zeta_{\gamma}\right)\right] \cdot \nabla_{\mathbf{r}_{\alpha}}\right. \\
& \quad+\frac{1}{M_{\alpha}}\left[\boldsymbol{\nabla}_{\boldsymbol{\rho}_{\alpha}} \ln \mathcal{F}_{\beta}^{(2)}\left(i \zeta_{\beta}\right)+\nabla_{\boldsymbol{\rho}_{\alpha}} \ln \mathcal{F}_{\gamma}^{(2)}\left(i \zeta_{\gamma}\right)\right] \cdot \boldsymbol{\nabla}_{\boldsymbol{\rho}_{\alpha}}-V_{\alpha}\left(\mathbf{r}_{\alpha}\right)+\frac{1}{\mu_{\alpha}} \boldsymbol{\nabla}_{\mathbf{r}_{\alpha}} \ln \mathcal{F}_{\beta}^{(2)}\left(i \zeta_{\beta}\right) \cdot \nabla_{\mathbf{r}_{\alpha}} \ln \mathcal{F}_{\gamma}^{(2)}\left(i \zeta_{\gamma}\right) \\
& \left.\quad+\frac{1}{M_{\alpha}} \boldsymbol{\nabla}_{\boldsymbol{\rho}_{\alpha}} \ln \mathcal{F}_{\beta}^{(2)}\left(i \zeta_{\beta}\right) \cdot \nabla_{\boldsymbol{\rho}_{\alpha}} \ln \mathcal{F}_{\gamma}^{(2)}\left(i \zeta_{\gamma}\right)\right] \varphi_{\alpha}^{(22)}\left(\mathbf{r}_{\alpha}, \boldsymbol{\rho}_{\alpha}\right)=O\left(1 / \rho_{\alpha}^{3}\right) .
\end{aligned}
$$

Using the same arguments we have used before, we may drop all the terms containing derivatives over $\rho_{\alpha}$ when looking for a solution in leading order. Then the equation for $\varphi_{\alpha}^{(22)}$ reduces to

$$
\left[\frac{1}{2 \mu_{\alpha}} \boldsymbol{\Delta}_{\mathbf{r}_{\alpha}}+i \frac{1}{\mu_{\alpha}} \mathbf{k}_{\alpha}^{(22)}\left(\boldsymbol{\rho}_{\alpha}\right) \cdot \boldsymbol{\nabla}_{\mathbf{r}_{\alpha}}-V_{\alpha}\left(\mathbf{r}_{\alpha}\right)\right] \varphi_{\alpha}^{(22)}\left(\mathbf{r}_{\alpha}, \boldsymbol{\rho}_{\alpha}\right)=0,
$$

with a local momentum

$$
\mathbf{k}_{\alpha}^{(22)}\left(\boldsymbol{\rho}_{\alpha}\right)=\mathbf{k}_{\alpha}+\sum_{\nu=\beta, \gamma} \frac{m_{\nu}}{m_{\beta \gamma}} k_{\nu}\left(\hat{\mathbf{k}}_{\nu}-\boldsymbol{\epsilon}_{\alpha \nu} \hat{\boldsymbol{\rho}}_{\alpha}\right) .
$$

If $V_{\alpha}$ is a pure Coulomb potential, $V_{\alpha}=V_{\alpha}^{C}$, then Eqs. (55), (70), (75), and (78) have the following solution:

$$
\varphi_{\alpha}^{(i j)}\left(\mathbf{r}_{\alpha}, \boldsymbol{\rho}_{\alpha}\right)=N_{\alpha}^{(i j)}\left(\boldsymbol{\rho}_{\alpha}\right) F\left[-i \eta_{\alpha}^{(i j)}\left(\boldsymbol{\rho}_{\alpha}\right), 1 ; i \zeta^{(i j)}\left(\boldsymbol{\rho}_{\alpha}\right)\right]
$$

Here, $i=1,2 ; j=1,2$; and $N_{\alpha}^{(i j)}\left(\boldsymbol{\rho}_{\alpha}\right)$ is defined as

$$
N_{\alpha}^{(i j)}\left(\boldsymbol{\rho}_{\alpha}\right)=e^{-\pi \eta_{\alpha}^{(i j)}\left(\boldsymbol{\rho}_{\alpha}\right) / 2} \Gamma\left[1+i \eta_{\alpha}^{(i j)}\left(\boldsymbol{\rho}_{\alpha}\right)\right]
$$

where $\quad \eta_{\alpha}^{(i j)}\left(\boldsymbol{\rho}_{\alpha}\right)=\left[z_{\beta} z_{\gamma} e^{2} \mu_{\alpha} / k_{\alpha}^{(i j)}\left(\boldsymbol{\rho}_{\alpha}\right)\right], \quad$ and $\quad \zeta^{(i j)}\left(\boldsymbol{\rho}_{\alpha}\right)$ $=k_{\alpha}^{(i j)}\left(\boldsymbol{\rho}_{\alpha}\right) r_{\alpha}-\mathbf{k}_{\alpha}^{(i j)}\left(\boldsymbol{\rho}_{\alpha}\right) \cdot \mathbf{r}_{\alpha}$. 
If $V_{\alpha}$ is not a pure Coulomb potential, then the differential equations above, which parametrically depend on $\boldsymbol{\rho}_{\alpha}$, should be solved numerically. Since all equations are of the twobody type, numerical methods are well developed and have been in use for a long time. They can be applied to solve the differential equations above as well. All the solutions found this way are valid in all directions of the asymptotic region $\Omega_{\alpha}$ except for singular directions.

Thus, returning to Eq. (38) we can claim that, having derived all four wave functions $\varphi_{\alpha(1)}^{(i j)}\left(\mathbf{r}_{\alpha}, \rho_{\alpha}\right), i, j=1,2$, we know the asymptotic behavior of the three-body incident wave of the scattering wave function of the first type in the asymptotic region $\Omega_{\alpha}$ up to the terms $O\left(1 / \rho_{\alpha}^{3}\right)$.

\section{GENERALIZED ASYMPTOTIC SCATTERING WAVE FUNCTION VALID IN ALL REGIONS $\Omega_{\nu}, \nu=\alpha, \beta, \gamma$}

Now we are in position to present a generalized asymptotic scattering wave function which satisfies the Schrödinger equation up to second order and which is valid in all the asymptotic regions:

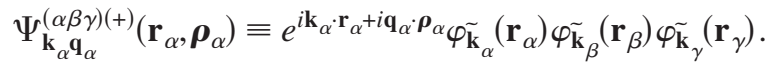

After substituting (82) into (3) and dropping the higher order terms we get

$$
\begin{aligned}
& \left\{E-T_{\mathbf{r}_{\alpha}}-T_{\vec{\rho}_{\alpha}}-V\right\}\left[e^{i \mathbf{k}_{\alpha} \cdot \mathbf{r}_{\alpha}+i \mathbf{q}_{\alpha} \cdot \boldsymbol{\rho}_{\alpha}}{\widetilde{\mathbf{k}_{\alpha}}}_{\alpha}\left(\mathbf{r}_{\alpha}\right) \varphi_{\mathbf{k}_{\beta}}\left(\mathbf{r}_{\beta}\right){\widetilde{\mathbf{k}_{\gamma}}}_{\gamma}\left(\mathbf{r}_{\gamma}\right)\right] \approx e^{i \mathbf{k}_{\alpha} \cdot \mathbf{r}_{\alpha}+i \mathbf{q}_{\alpha} \cdot \boldsymbol{\rho}_{\alpha}}{\widetilde{\mathbf{k}_{\beta}}}_{\beta}\left(\mathbf{r}_{\beta}\right) \varphi_{\mathbf{k}_{\gamma}}\left(\mathbf{r}_{\gamma}\right)\left[\frac{\Delta_{\mathbf{r}_{\alpha}}}{2 \mu_{\alpha}}+\frac{i \widetilde{\mathbf{k}}_{\alpha} \cdot \nabla_{\mathbf{r}_{\alpha}}}{\mu_{\alpha}}-V_{\alpha}\right] \varphi_{\mathbf{k}_{\alpha}}\left(\mathbf{r}_{\alpha}\right)
\end{aligned}
$$

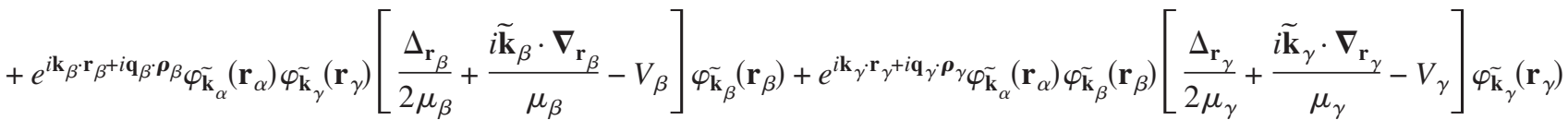

$$
=\left\{\begin{array}{c}
O\left(\frac{1}{r_{\alpha}^{2}}, \frac{1}{r_{\beta}^{2}}, \frac{1}{r_{\gamma}^{2}}\right), \mathbf{r}_{\alpha}, \mathbf{r}_{\beta}, \mathbf{r}_{\gamma} \in \Omega_{0} \\
O\left(\frac{1}{r_{\beta}^{2}}, \frac{1}{r_{\gamma}^{2}}\right), \mathbf{r}_{\beta}, \mathbf{r}_{\gamma} \in \Omega_{\alpha} \\
O\left(\frac{1}{r_{\alpha}^{2}}, \frac{1}{r_{\gamma}^{2}}\right), \mathbf{r}_{\alpha}, \mathbf{r}_{\gamma} \in \Omega_{\beta} \\
O\left(\frac{1}{r_{\alpha}^{2}}, \frac{1}{r_{\beta}^{2}}\right), \mathbf{r}_{\alpha}, \mathbf{r}_{\beta} \in \Omega_{\gamma}
\end{array}\right.
$$

\section{CONCLUSION}

We derived the three-body asymptotic incident wave, which satisfies the Schrödinger equation in the asymptotic region $\Omega_{\nu}, \nu=\alpha, \beta, \gamma$ up to terms of order $1 / \rho_{\nu}^{3}$. This asymptotic incident wave gives the leading asymptotic terms of the three-body scattering wave function of the first type and is an extension of the asymptotic wave function derived in $[2,3]$. Equivalently, similar wave functions satisfy the Schrödinger equation up to $O\left(1 / \rho_{\nu}^{3}\right), \nu=\beta, \gamma$. It is worth mentioning that the asymptotic solution satisfying the Schrödinger equation, in the asymptotic region $\Omega_{\nu}$ up to the $O\left(1 / \rho_{\nu}^{2}\right)$, can be found analytically $[2,3]$. To find an asymptotic solution satisfying the Schrödinger equation in $\Omega_{\nu}$ up to terms of $O\left(1 / \rho_{\nu}^{3}\right)$ we need to solve two-body type differential equations numerically. The next order term in the asymptotic three-body scattering wave function represents the outgoing 3 particles $\rightarrow 3$ particles scattered wave and has been given in [7].

The resulting asymptotic solution provides extended boundary conditions in all the asymptotic regions and can be used in the direct numerical solution of the Schrödinger equation or in approximate perturbation calculations as a leading asymptotic term of the three-body scattering wave function.

\section{ACKNOWLEDGMENTS}

This work was supported by the U.S. DOE under Grant No. DE-FG03-93ER40773, by NSF Grant No. PHY0140343, and the Australian Research Council. 
[1] L. D. Faddeev and S. P. Merkuriev, Quantum Scattering Theory for Several Particle Systems (Kluwer Academic Publishers, Dordrecht, 1993).

[2] E. O. Alt and A. M. Mukhamedzhanov, Phys. Rev. A 47, 2004 (1993).

[3] A. M. Mukhamedzhanov and M. Lieber, Phys. Rev. A 54, 3078 (1996).

[4] A. S. Kadyrov, A. M. Mukhamedzhanov, and A. T. Stelbovics, Phys. Rev. A 67, 024702 (2003).

[5] A. S. Kadyrov, A. M. Mukhamedzhanov, A. T. Stelbovics, I. Bray, and F. Pirlepesov, Phys. Rev. A 68, 022703 (2003).

[6] A. S. Kadyrov, A. M. Mukhamedzhanov, A. T. Stelbovics, and I. Bray, Phys. Rev. Lett. 91, 253202 (2003).
[7] A. S. Kadyrov, A. M. Mukhamedzhanov, A. T. Stelbovics, and I. Bray, Phys. Rev. A 70, 062703 (2004).

[8] L. D. Landau and E. M. Lifshitz, Quantum Mechanics (Nonrelativistic Theory), 3rd ed., Course of Theoretical Physics Vol. 3 (Pergamon Press, Oxford, 1985).

[9] A. Kratzer and W. Franz, Transzendente Funktionen, 2nd ed. (Akadem. Verlagsgesellschaft, Leipzig, 1963).

[10] I. S. Gradshteyn and I. M. Ryzhik, Tables of Integrals, Series and Products (Academic Press, San Diego, 1980).

[11] P. J. Redmond, described in [12] (unpublished).

[12] L. Rosenberg, Phys. Rev. D 8, 1833 (1973).

[13] C. R. Garibotti and J. E. Miraglia, Phys. Rev. A 21, 572 (1980). 